\title{
Molecular Abundances in the Atmosphere of the T Dwarf Gl 229B
}

\author{
D. Saumon \\ Department of Physics and Astronomy, Vanderbilt University, Nashville, TN 37235 \\ dsaumon@cactus.phy. vanderbilt.edu \\ T.R. Geballe \\ Gemini North Observatory, 670 North A'ohoku Place, Hilo, HI 96720 \\ S.K. Leggett \\ Joint Astronomy Center, 660 North A'ohoku Place, Hilo, HI 96720 \\ M.S. Marley \\ Department of Astronomy, New Mexico State University, Las Cruces, NM 88003 \\ R.S. Freedman \\ The Space Physics Research Institute, NASA Ames Research Center \\ Moffett Field, CA 94035 \\ K. Lodders, B. Fegley, Jr. \\ Planetary Chemistry Laboratory, Department of Earth 8 Planetary Science \\ Washington University, St-Louis, MO 63130 \\ and \\ S.K. Sengupta \\ Department of Physics and Astronomy, Vanderbilt University, Nashville, TN 37235
}

\begin{abstract}
We present new, high resolution, infrared spectra of the T dwarf Gliese 229B in the $J, H$, and $K$ bandpasses. We analyze each of these as well as previously published spectra to determine its metallicity and the abundances of $\mathrm{NH}_{3}$ and $\mathrm{CO}$ in terms of the surface gravity of Gl 229B, which remains poorly constrained. The metallicity increases with increasing gravity and is below the solar value unless Gl 229B is a high-gravity brown dwarf with $\log g(\operatorname{cgs}) \approx 5.5$. The $\mathrm{NH}_{3}$ abundance is determined from both the $H$ and the $K$ band spectra which probe two different levels in the atmosphere. We find that the abundance from the $K$ band data is well below that expected from chemical equilibrium, which we interpret as strong evidence for dynamical transport of $\mathrm{NH}_{3}$ in the atmosphere. This is consistent with the previous detection of $\mathrm{CO}$ and provides additional constraints on the dynamics of the atmosphere of this $\mathrm{T}$ dwarf.
\end{abstract}

Subject headings: stars: individual (Gl 229B) — stars: brown dwarfs — stars: atmospheres — stars: abundances 


\section{Introduction}

Gliese 229B is not only the first brown dwarf recognized as genuine (Nakajima et al. 1995; Oppenheimer et al. 1995), but it is also the brightest and best-studied $\mathrm{T}$ dwarf known. With an effective temperature of $T_{\text {eff }} \sim 950 \mathrm{~K}$, it lies squarely between the latest $\mathrm{L}$ dwarfs $\left(T_{\text {eff }} \sim 1500 \mathrm{~K}\right.$, Kirkpatrick et al. (1998)) and the giant planets of the solar system $\left(T_{\text {eff }} \sim 100 \mathrm{~K}\right)$. Indeed, its near infrared spectrum shows the strong $\mathrm{H}_{2} \mathrm{O}$ absorption bands characteristic of very-low mass stars and the strong $\mathrm{CH}_{4}$ bands seen in the spectra of Jupiter, Saturn and Titan. The transitional nature of the spectrum of Gl 229B is remarkable and hints at the spectral appearance of extrasolar giant planets which have effective temperatures in the range $200-1600 \mathrm{~K}$ (Guillot 1999).

A wealth of data on Gl 229B has accumulated since its discovery five years ago. Broad band photometry from $R$ through $N$ and an accurate parallax (Matthews et al. 1996; Golimowski et al. 1998; Leggett et al. 1999; Perryman et al. 1997) allow an accurate determination of its bolometric luminosity. Spectroscopic observations (Oppenheimer et al. 1998; Geballe et al. 1996; Schultz et al. 1998) covering the range from 0.8 to $5.0 \mu \mathrm{m}$ have revealed a very rapidly declining flux shortward of $1 \mu \mathrm{m}$, the unmistakable presence of $\mathrm{CH}_{4}$, $\mathrm{H}_{2} \mathrm{O}$, and $\mathrm{Cs}$, and demonstrated the absence of the $\mathrm{CrH}, \mathrm{FeH}, \mathrm{VO}$ and $\mathrm{TiO}$ features characteristic of late $\mathrm{M}$ and early L dwarfs. Finally, Noll, Geballe \& Marley (1997) and Oppenheimer et al. (1998) have detected $\mathrm{CO}$ with an abundance well above the value predicted by chemical equilibrium, a phenomenon also seen in the atmosphere of Jupiter.

Model spectra for Gl 229B (Marley et al. 1996; Allard et al. 1996; Tsuji et al. 1996b) reproduce the overall energy distribution fairly well and all agree that 1) $T_{\text {eff }} \sim 950 \mathrm{~K}, 2$ ) compared to gaseous molecular opacity, the dust opacity is small if not negligible in the infrared, 3) the gravity of Gl 229B is poorly constrained at present. The rapid decline of the flux at wavelengths shortward of $1 \mu \mathrm{m}$ is interpreted as caused by an absorbing haze of complex hydrocarbons (Griffith, Yelle \& Marley 1998) or alternatively by the pressure-broadened red wing of the K I resonance doublet at $0.77 \mu \mathrm{m}$ (Tsuji, Ohnaka \& Aoki 1999; Burrows, Marley \& Sharp 1999).
In this paper, we present new high-resolution spectra in the $J, H$, and $K$ bands. With the inclusion of the "red" spectrum of Oppenheimer et al. (1998), we analyze each part of the spectrum separately to obtain independent measures of the $\mathrm{H}_{2} \mathrm{O}$ abundance of Gl 229B - broadly interpreted as the metallicity index - to detect for the first time the presence of $\mathrm{NH}_{3}$ in its spectrum, and to estimate the $\mathrm{NH}_{3}$ abundance at two different depths in the atmosphere. Our results are expressed in terms of the surface gravity which cannot be determined from the data presented here. Nevertheless, we identify a reduced set of acceptable combinations of $T_{\text {eff }}$ and gravity, using the observed bolometric luminosity of Gl 229B (Leggett et al. 1999).

The observations and the near infrared spectra are presented in $\S 2$. Section 3 shows how an accurate parallax, a well-sampled spectral energy distribution and evolutionary models greatly reduce the possible range of combinations of $T_{\text {eff }}$ and gravity without having to resort to spectrum fitting. The synthetic spectrum calculation and our method of analysis are described in $\S 4$. The results concerning several molecules of interest which are at least potentially detectable are presented in $\S 5$, followed by a discussion in $\S 6$. Finally, a summary of the results and directions for future study are given in $\S 7$.

\section{Spectroscopic observations}

Spectra of Gl 229B in selected narrow intervals in the $J, H$, and $K$ windows were obtained at the $3.8 \mathrm{~m}$ United Kingdom Infrared Telescope (UKIRT) in 1998 January, using the facility spectrometer CGS4 (Mountain et al. 1990) and its 150 $\mathrm{l} / \mathrm{mm}$ grating. Details of the observations are provided in Table 1 . These are among the highest resolution spectra obtained of any $\mathrm{T}$ dwarf.

The spectra were obtained in the standard stare/nod mode with the $1.2^{\prime \prime}$ wide slit of the spectrometer oriented at a position angle of $45^{\circ}$, nearly perpendicular to the line connecting Gl 229A and Gl 229B. The southward-going diffraction spike of Gl 229A together with scattered light from that star, which is 10 magnitudes brighter than Gl 229B, contaminated the array rows near and to the southwest of those containing the spectrum of Gl 229B. The contamination on the Gl 229B rows was determined by interpolation and was subtracted; 
typically it was comparable or somewhat smaller than the signal from Gl 229B. In order to remove telluric absorption features, spectra of the A0V star BS 1849 were measured just prior to Gl 229B. In all cases the match in airmasses was better than five percent and hence in the ratioed spectra residual telluric features are small compared to the noise level. Wavelength calibration was achieved by observations of arc lamps and is in all cases better than one part in $10^{4}(2 \sigma)$.

The spectra shown in this paper have been slightly smoothed, so that the resolving powers are lower than those in Table 1 by approximately 25 percent. They also have been rebinned to facilitate coadding like spectra and joining adjacent spectral regions. The error bars can be judged by the point-to-point variations in featureless portions of the spectra, the signal-to-noise ratios at the continuum peaks are approximately 40 in the $K$ band, 25 in the $H$ band, and 30 in the $J$ band. The flux calibration of each spectrum is approximate as no attempt was made to match the photometry of Gl 229B.

While we identify the spectra by their corresponding standard photometric infrared bandpass, their wavelength coverages are much narrower than the $J H K$ filters and typically corresponds to the peak flux of Gl 229B in each bandpass.

In the $J$ band spectrum (Fig. 1), nearly all features are caused by $\mathrm{H}_{2} \mathrm{O}$. The short wavelength end of the spectrum shows the red side of a $\mathrm{CH}_{4}$ band, which is responsible for the features seen shortward of $\sim 1.215 \mu \mathrm{m}$. Two lines of neutral potassium are easily detected near $1.25 \mu \mathrm{m}$. No other lines of alkali metals fall within the wavelength coverage of our observations. The $H$ band spectrum (Fig. 2) is relatively rich in molecular opacity sources. All the features seen in the spectrum are either due to $\mathrm{H}_{2} \mathrm{O}(\lambda \lesssim 1.59 \mu \mathrm{m})$ or part of a very strong $\mathrm{CH}_{4}$ absorption band $(\lambda \gtrsim 1.6 \mu \mathrm{m})$. Features seen between 1.59 and $1.6 \mu \mathrm{m}$ cannot presently be ascribed with certainty and are due to either $\mathrm{H}_{2} \mathrm{O}$ or $\mathrm{CH}_{4}$. While the opacities of $\mathrm{NH}_{3}$ and $\mathrm{H}_{2} \mathrm{~S}$ are not negligible in this part of the spectrum, neither molecule forms distinctive spectral features. Their presence cannot be directly ascertained from these data, mainly because their opacity is weaker than that of $\mathrm{H}_{2} \mathrm{O}$ and $\mathrm{CH}_{4}$ and because of significant pressure broadening (see
§5.3). The $K$ band flux emerges in an opacity window between a strong $\mathrm{H}_{2} \mathrm{O}$ band and a strong $\mathrm{CH}_{4}$ band (Fig. 3). Spectral features are caused by $\mathrm{H}_{2} \mathrm{O}$ at shorter wavelengths $(\lambda \leq 2.11 \mu \mathrm{m})$ and by $\mathrm{CH}_{4}$ at longer wavelengths $(\lambda \geq 2.105 \mu \mathrm{m})$. Several distinctive features of $\mathrm{NH}_{3}$ are expected at the blue end of this spectrum and models predict a single absorption feature of $\mathrm{H}_{2} \mathrm{~S}$ at $2.1084 \mu \mathrm{m}$.

All features seen in Figures $1-3$ are unresolved blends of numerous molecular transitions. A spectral resolution at least 10 times higher would be required to resolve the intrinsic structure of the spectrum of Gl 229B.

\section{Effective temperature and gravity}

While synthetic spectra have been fairly successful at reproducing the unusual spectrum of Gl 229B (Marley et al. 1996; Allard et al. 1996; Tsuji et al. 1996b), the entire spectral energy distribution has not yet been modeled satisfactorily. Limitations in the opacity databases are partly responsible for the remaining discrepancies between synthetic spectra and the data (see $\S 4.2$ ). These shortcomings have impeded the determination of $T_{\text {eff }}$ and of the gravity $g$ in particular. On the other hand, the bolometric luminosity of Gl 229B is now well determined. Combining spectroscopic and photometric data from 0.82 to $10 \mu \mathrm{m}$ with the parallax, Matthews et al. (1996) found $L=6.4 \times 10^{-6} L_{\odot}$. With new JHKL' photometry, Leggett et al. (1999) found $L=6.6 \pm 0.6 \times 10^{-6} L_{\odot}$. A recalibration using the HST photometry of Golimowski et al. (1998) gives $L=6.2 \pm 0.55 \times 10^{-6} L_{\odot}$. Evolutionary models (Burrows et al. 1997) allow us to find a family of $\left(T_{\mathrm{eff}}, g\right)$ models with a given $L_{\mathrm{bol}}$. Figure 4 shows the cooling tracks of solar metallicity brown dwarfs in terms of the surface parameters $T_{\text {eff }}$ and $g$. Models with the bolometric luminosity of Gl 229B fall within the band running through the center of the figure. Using a very conservative lower limit for the age of Gl 229A of $0.2 \mathrm{Gyr}$ (Nakajima et al. 1995), we find $T_{\text {eff }}=950 \pm 80 \mathrm{~K}$. On the other hand, the gravity remains poorly determined with $\log g\left(\mathrm{~cm} / \mathrm{s}^{2}\right)=5 \pm 0.5$, corresponding to a mass range of $0.015-0.07 M_{\odot}$. While the upper range is very close to the lower main sequence mass limit, Gl 229B's status as a brown dwarf is secure. A star at the edge of the main sequence 
would be much hotter with $T_{\text {eff }} \sim 1800 \mathrm{~K}$; well outside of Fig. 4.

In the remainder of this paper, the discussion focuses on three atmosphere models which span the range of allowed solutions (Table 2 and Fig. $4):\left(T_{\text {eff }}(\mathrm{K}), \log g(\mathrm{cgs})\right)=(870,4.5),(940,5.0)$ and $(1030,5.5)$, which we label models A, B, and $\mathrm{C}$, respectively. These constraints on $T_{\text {eff }}$ and $g$ from cooling sequences are quite firm. We find the same result, within the error bar on $L_{\mathrm{bol}}$, from several cooling sequences which predate Burrows et al. (1997). The latter were computed with different input physics such as the equation of state and surface boundary conditions derived from grey and non-grey atmosphere models using several opacity tabulations.

In section 5, we show that these three models can fit the spectra only if they have different metallicities, ranging from $[\mathrm{M} / \mathrm{H}]=-0.1$ to -0.5 . The evolution of brown dwarfs is sensitive to the metallicity through the atmospheric opacity which controls the rate of cooling. We find that for the range of interest here, the effect of a reduced metallicity on our determination of $T_{\text {eff }}, g$, and the cooling age is smaller but comparable to that of the uncertainty on the value of $L_{\mathrm{bol}}$. We choose to ignore it for simplicity.

\section{Method of analysis}

\subsection{Model atmospheres and spectra}

Our analysis is based on the atmosphere models of brown dwarfs and extrasolar giant planets described in Burrows et al. (1997). Briefly, the atmospheres are in radiative/convective equilibrium and the equation of radiative transfer is solved with the k-coefficient method. The chemical equilibrium is treated as in Burrows et al. (1997). Gas phase opacities include Rayleigh scattering, the collision-induced opacity of $\mathrm{H}_{2}$ and the molecular opacities of $\mathrm{H}_{2} \mathrm{O}, \mathrm{CH}_{4}, \mathrm{NH}_{3}, \mathrm{H}_{2} \mathrm{~S}, \mathrm{PH}_{3}$, and $\mathrm{CO}$, as well as the continuum opacities of $\mathrm{H}^{-}$and $\mathrm{H}_{2}^{-}$. The molecular line opacity database is described in more detail in $\S 4.2$. Atomic line opacity is not included. Because of the relatively large gravity of Gl 229B, pressure broadening of the molecular lines plays an important role in determining the $(T, P)$ profile of the atmosphere and in shaping the spectrum. The line-by-line broadening theory we use is described in Burrows et al. (1997). The strong continuum opacity source responsible for the rapid decrease of the flux of Gl 229B shortward of $1.1 \mu \mathrm{m}$ is included following the haze model of Griffith et al. (1998). Details of the haze model and of our fitting procedure are given in $\S 5.1$. The $(T, P)$ structures of these atmosphere models are shown in Fig. 5. The profiles intersect each other because both $T_{\text {eff }}$ and the gravity vary between the models. The inflexion point at $\log T \sim 3.25$ signals the top of the convection zone.

Using the same monochromatic opacities used to compute the k-coefficients, high-resolution synthetic spectra are generated from the atmospheric structures by solving the radiative transfer equation with the Feautrier method on a frequency grid with $\Delta \nu=0.1 \mathrm{~cm}^{-1}$. Spectra with resolution lower than $\Delta \nu \gtrsim 1 \mathrm{~cm}^{-1}$ can then be generated for comparison with data.

An unusual aspect of $\mathrm{T}$ dwarf atmospheres is the great variation of the opacity with wavelength. These atmospheres are strongly non-grey and the near infrared spectrum is sculpted by strong absorption bands of $\mathrm{H}_{2} \mathrm{O}$ and $\mathrm{CH}_{4}$. Most of the flux emerges in a small number of relatively transparent opacity windows. The concept of photosphere becomes rather useless since the level at which the spectrum is formed depends strongly on the wavelength. Figure 6 shows the depth of the "photosphere" $\left(\tau_{\nu}=2 / 3\right)$ in both temperature and pressure as a function of wavelength for model $\mathrm{B}\left(T_{\mathrm{eff}}=940 \mathrm{~K}, \log g=5\right)$. In the $Z, J$, and $H$ bands, and, to a lesser extent, in the $K$ and $M$ bands, the atmosphere is very transparent and can be probed to great depths. For $\lambda \gtrsim 25 \mu \mathrm{m}$, the spectral energy distribution approaches a Planck function with $T \sim 500 \mathrm{~K}$.

Figure 6 reveals that spectroscopy between 0.8 and $12 \mu \mathrm{m}$ can probe the atmosphere from $T \sim$ $500 \mathrm{~K}$ down to a depth where $T \sim 1600 \mathrm{~K}$, corresponding to a range of 6 pressure scale heights! This provides an exceptional opportunity to study the physics of the atmosphere of a brown dwarf over an extended vertical range. The top of the convection zone for model B (located at $T=$ $1860 \mathrm{~K})$ is below the "photosphere" at all wavelengths and is not directly observable, however. 


\subsection{Limitations to this study}

Given the range of acceptable values of $T_{\text {eff }}$ and $g(\S 3)$, we can determine the metallicity of Gl $229 \mathrm{~B}$ and the abundance of several key molecules by fitting synthetic spectra to the observations, for each of the three models. The precision of our results is determined by the reliability of the models, the noise level in the data and, most significantly, by the limitations of the molecular line lists used to compute their opacities. The latter point requires a detailed discussion.

The opacities of $\mathrm{CH}_{4}$ and $\mathrm{NH}_{3}$ are computed from line lists obtained by combining the HITRAN (Rothman et al. 1998) and GEISA (Husson et al. 1994) databases, which are complemented with recent laboratory measurements and theoretical calculations. Further details are provided in Burrows et al. (1997). The resulting line lists for these two molecules are very nearly complete for $T<300 \mathrm{~K}$, and their degree of completeness decreases rapidly at higher temperatures where absorption from excited level become important. Furthermore, the line list of $\mathrm{CH}_{4}$ is limited to $\lambda>1.584 \mu \mathrm{m}$. We extend the $\mathrm{CH}_{4}$ opacity to shorter wavelengths with the laboratory measurements of Strong et al. (1993) which provide the absorption coefficient averaged over intervals of $5 \mathrm{~cm}^{-1}$ between 1 and $5 \mu \mathrm{m}$ at $T=300 \mathrm{~K}$. We use Strong et al. (1993) opacities for $1<\lambda<1.82 \mu \mathrm{m}$ and the line list for $\lambda>1.82 \mu \mathrm{m}$. This puts the transition from one tabulation to the other in a strong $\mathrm{H}_{2} \mathrm{O}$ absorption band and obliterates any discontinuity in $\mathrm{CH}_{4}$ opacity at the transition. For $\lambda<1 \mu \mathrm{m}$, the tabulation of Karkoschka (1994) gives the absorption coefficient of $\mathrm{CH}_{4}$ determined from spectroscopic observations of the giant planets at $0.0004 \mu \mathrm{m}$ intervals. Because of the low temperatures found in the atmospheres of giant planets, the Karkoschka $\mathrm{CH}_{4}$ opacities are appropriate for $T \lesssim 200 \mathrm{~K}$. To our knowledge, this compilation of $\mathrm{NH}_{3}$ and $\mathrm{CH}_{4}$ opacity is the most complete presently available.

As can be seen in Figure 6, the temperature in the atmosphere of Gl 229B is everywhere greater than $300 \mathrm{~K}$. For $\mathrm{CH}_{4}$, we compute temperaturedependent line opacity (which is incomplete above $300 \mathrm{~K}$ ) from the population of excited levels determined by the Boltzmann formula for $\lambda>1.82 \mu \mathrm{m}$, and use temperature independent opacity at shorter wavelengths. While the synthetic spec- tra computed reproduce the fundamental band of $\mathrm{CH}_{4}$ very well (centered at $\lambda=3.4 \mu \mathrm{m}$ ), the match with the 1.6 and $2.3 \mu \mathrm{m}$ bands is rather poor. Even though $\mathrm{CH}_{4}$ is a very prominent molecule in the spectrum of Gl 229B, the current knowledge of its opacity is not adequate for a quantitative analysis of its spectral signature. For this reason, we have essentially ignored the regions in our spectra where $\mathrm{CH}_{4}$ is prominent. Unfortunately, this prevents us from estimating the abundance of $\mathrm{CH}_{4}$ and therefore of carbon - in Gl 229B.

Ammonia shows significant absorption in both the $H$ and $K$ band spectra. The line list for $\mathrm{NH}_{3}$ starts at $\lambda>1.415 \mu \mathrm{m}$. Because the line list does not include transitions from highly excited levels which occur at $T>300 \mathrm{~K}$, the $\mathrm{NH}_{3}$ opacity we compute at a given wavelength is strictly a lower limit to the actual opacity.

Except for the collision-induced absorption by $\mathrm{H}_{2}$, the most important molecular absorber in Gl $229 \mathrm{~B}$ is $\mathrm{H}_{2} \mathrm{O}$, for which the opacity is now relatively well understood. We use the most recent and most extensive ab initio line list (Partridge \& Schwenke (1997), $3 \times 10^{8}$ transitions). This line list is essentially complete for $T \lesssim 3000 \mathrm{~K}$. As a demonstration of the equality of this database, we find that the $\mathrm{H}_{2} \mathrm{O}$ features computed with this line list correspond extremely well in frequency with the observed features of Gl 229B (e.g. Figs. 7 to 9 ). However, we find noticeable discrepancies in the relative strengths of $\mathrm{H}_{2} \mathrm{O}$ features which we attribute to the calculated oscillator strength of the transitions (§5.1). This effect can also be seen in Fig. 1c of Griffith et al. (1998). At high resolution, the distribution of molecular transitions in frequency and strength is nearly random, and the inaccuracies in oscillator strengths we have found limit the accuracy of model fitting in a fashion similar to noise. This "opacity noise" is at least as significant as the noise intrinsic to our data.

\section{Results from fitting the spectra}

We have constructed a grid of synthetic spectra for the three models shown in Fig. 4 with metallicity $-0.7 \leq[\mathrm{M} / \mathrm{H}] \leq 0.1$ in steps of 0.1 . We use these modeled spectra to fit four distinct spectral regions (the "red", $J, H$, and $K$ spectra) separately to determine the metallicity as a function of gravity with an internal precision of \pm 0.1 dex. 
For the purpose of fitting the data, the synthetic spectra were renormalized to the observed flux at a selected wavelength in each spectral region.

The model spectra show some distortions in the overall shape of the spectrum which are probably due to remaining uncertainties in the $(P, T)$ profile of the atmosphere, the inadequate $\mathrm{CH}_{4}$ opacities, and possible effects of dust opacity. Considering the additional problems with the strength of the $\mathrm{H}_{2} \mathrm{O}$ features, we elected to do all fits "by eye," except where otherwise noted. We discuss the fitting of each spectral interval below. In the interest of brevity, we present a detailed discussion of fits obtained only with the model of intermediate gravity (model B). The best fits obtained with models $\mathrm{A}$ and $\mathrm{C}$ are very nearly identical to those with model B. The results are summarized in Table 2.

\subsection{The "red" spectrum}

The "red" spectrum extends from 0.83 to $\sim$ $1 \mu \mathrm{m}$ and is formed deep in the atmosphere where $1100<T<1500 \mathrm{~K}$. The spectra of Schultz et al. (1998) and Oppenheimer et al. (1998) reveal two lines of Cs I (at 0.852 and $0.894 \mu \mathrm{m}$ ) and a strong $\mathrm{H}_{2} \mathrm{O}$ band but not the bands of $\mathrm{TiO}$ and VO common to late $\mathrm{M}$ dwarfs and early $\mathrm{L}$ dwarfs (Fig. 7). Refractory elements, such as Ti, Fe, V, Ca, and $\mathrm{Cr}$, are expected to be bound in condensed compounds in a low-temperature atmosphere such as that of $\mathrm{Gl} 229 \mathrm{~B}$ and therefore are not available to form molecular bands (Fegley \& Lodders 1996; Marley et al. 1996; Burrows \& Sharp 1999). With the exception of the strong, unidentified feature at $0.9874 \mu \mathrm{m}$, all features between 0.89 and $1.0 \mu \mathrm{m}$ can be attributed to $\mathrm{H}_{2} \mathrm{O}$. An overlap of a weak band of $\mathrm{H}_{2} \mathrm{O}$ and a weaker $\mathrm{CH}_{4}$ band causes the small depression at $0.894 \mu \mathrm{m}$ which was tentatively tentatively attributed to $\mathrm{CH}_{4}$ by $\mathrm{Op}$ penheimer et al. (1998) and Schultz et al. (1998). Features below $0.89 \mu \mathrm{m}$ cannot be identified at present. The two Cs I lines are not included in our model.

The flux from Gl $229 \mathrm{~B}$ is also observed to decrease very rapidly toward shorter wavelengths (Schultz et al. 1998; Oppenheimer et al. 1998; Golimowski et al. 1998), which, in the absence of the strong $\mathrm{TiO}$ and $\mathrm{VO}$ bands, is evidently caused by the presence of a missing source of opacity in the atmosphere. Spectra computed with molecular opacities only (but excluding $\mathrm{TiO}, \mathrm{VO}, \mathrm{FeH}$, etc) predict visible fluxes which are grossly overestimated (Griffith et al. 1998) but the detailed sequence of absorption features of the spectrum are well reproduced, indicating that the short wavelength flux is suppressed by a smooth opacity source.

We fit the red spectrum of Gl 229B between 0.82 and $1.15 \mu \mathrm{m}$ to obtain the metallicity. We have recalibrated the published spectrum (Oppenheimer et al. 1998; Geballe et al. 1996; Leggett et al. 1999) using the HST photometry of Golimowski et al. (1998). We model the continuum opacity with a layer of condensates following the approach of Griffith et al. (1998). Condensates are expected in the atmosphere of Gl 229B on the basis of chemical equilibrium calculations (Lodders 1999; Burrows \& Sharp 1999) and can provide the required opacity. Alternatively, Tsuji, Ohnaka \& Aoki (1999) and Burrows, Marley, \& Sharp (1999) attribute this rapid decline to the pressure broadened red wing of the $0.77 \mu \mathrm{m} \mathrm{K} \mathrm{I} \mathrm{resonance} \mathrm{dou-}$ blet. The first optical spectrum of a $\mathrm{T}$ dwarf (SDSS 1624+0029) shows that the latter explanation is correct (Liebert et al. 2000). The nature of this opacity source is not very important for the determination of the metallicity, however, as long as the proper continuum opacity background is present in the calculation.

The dust opacity is computed with the Mie theory of scattering by spherical particles and is determined by the vertical distribution of the particles, their grain size distribution, and the complex index of refraction of the condensate. The cloud model of Griffith et al. (1998) is described by 1) the vertical density profile of the condensate, taken as:

$$
n_{d}=A P \text {, }
$$

where $n_{d}$ is the number density of condensed particles, $P$ is the ambient gas pressure, and the cloud layer is bound by

$$
P_{\text {top }} \leq P \leq 100 \text { bar; }
$$

2) the size distribution of the particles

$$
f(d)=\frac{d}{d_{0}} \exp \left[\frac{\ln \left(d / d_{0}\right)}{\ln \sigma}\right]^{2},
$$

where $d$ is the diameter of the particles; and 3) the complex index of refraction of the condensate

$$
n(\lambda)=1.65+i n_{i}(\lambda) .
$$


The parameters $P_{\mathrm{top}}, A, d_{0}, \sigma$, the function $n_{i}(\lambda)$, and the metallicity of the atmosphere are free parameters.

Such a multi-parameter fit of the observed spectrum is not unique. Furthermore, arbitrarily good fits of the "continuum" flux level can be obtained by adjusting the imaginary part of the index of refraction since its wavelength dependence is weakly constrained a priori. Our results for the three models are qualitatively similar to those of Griffith et al. (1998). Typical values of the fitted dust parameters are $A=415 \mathrm{~cm}^{-3} \mathrm{bar}^{-1}, P_{\mathrm{top}}=0.5 \mathrm{bar}$, $d_{0}=0.21 \mu \mathrm{m}$, and $\sigma=1.3$, with the imaginary part of the index of refraction decreasing from $n_{i}=1.70$ at $0.8 \mu \mathrm{m}$ to 0.01 at $1.10 \mu \mathrm{m}$. These parameters applied to model $\mathrm{B}$ with a metallicity of $[\mathrm{M} / \mathrm{H}]=-0.3$ result in the fit shown in Figure 7.

The metallicity of the atmosphere $[\mathrm{M} / \mathrm{H}]$ is largely independent of the dust parameters, however, as it is constrained by the amplitude of the features in the $\mathrm{H}_{2} \mathrm{O}$ band which we fit between 0.925 and $0.98 \mu \mathrm{m}$. A larger metallicity results in a larger amplitude of the features inside the band. We obtain the same value of $[\mathrm{M} / \mathrm{H}]$ as long as a good fit of the "continuum" flux level is obtained, regardless of the particular values of dust parameters. The lower panel of Fig. 7 clearly shows differences in the relative strengths of the absorption features in the $\mathrm{H}_{2} \mathrm{O}$ band between the synthetic and observed spectra. Similar differences also occur in the $J, H$, and $K$ bands. The same differences are found for all three atmospheric profiles and point to inaccuracies in the oscillator strength of the ab initio line list of $\mathrm{H}_{2} \mathrm{O}$ (Partridge \& Schwenke 1997). The metallicity is fitted to give the best overall fit of these features with a precision of \pm 0.1 dex.

Strictly speaking, this procedure gives the $\mathrm{H}_{2} \mathrm{O}$ abundance, or $[\mathrm{O} / \mathrm{H}]$ rather than $[\mathrm{M} / \mathrm{H}]$. For solar metallicity, the condensation of silicates deep in the atmosphere of Gl 229B will reduce the amount of oxygen available to form $\mathrm{H}_{2} \mathrm{O}$ by $\sim 15 \%$ (Fegley \& Prinn 1988) which implies that

$$
[\mathrm{M} / \mathrm{H}]=[\mathrm{O} / \mathrm{H}]+0.07 .
$$

The correction, which decreases for subsolar metallicities, is smaller than our fitting uncertainty and will be ignored hereafter.

\section{2. $J$ band spectrum}

The $J$ band spectrum probes the most transparent window of the spectrum of Gl $229 \mathrm{~B}$ and is formed at great depth where $1500 \leq T \leq 1600 \mathrm{~K}$ and $P \sim 30$ bar (Fig. 6). Our spectrum contains almost exclusively $\mathrm{H}_{2} \mathrm{O}$ features, with the exception of $\mathrm{CH}_{4}$ absorption for $\lambda \lesssim 1.215 \mu \mathrm{m}$ and of two prominent K I lines (Fig. 1). Since we have elected to ignore $\mathrm{CH}_{4}$ bands and our synthetic spectra do not include alkali metal lines, we fit the $J$ spectrum between 1.215 and $1.298 \mu \mathrm{m}$ to determine the metallicity from the depth of the $\mathrm{H}_{2} \mathrm{O}$ absorption features. Figure 8 shows the effect of the metallicity on the spectrum (top panel) and our best fit (bottom panel) for model B. The flux level in the $J$ spectrum varies by a factor of 3 and our best fit shows distortions in the general shape of the spectrum. The distortions may be caused by a combination of uncertainties in the $(T, P)$ profile of the atmosphere, problems with the $\mathrm{H}_{2} \mathrm{O}$ opacities or a small amount of dust opacity in the infrared spectrum of Gl 229B (not modeled). Since the fit is based on the depth of the features, we ignore these distortions and fit the logarithm of the flux rather than the flux, as shown in Fig. 8. As in the red spectrum ( $\$ 5.1$ ), we find a remarkable correspondence of spectral features between the observed and modeled spectra although the model spectrum is somewhat less successful at reproducing the relative strengths of the $\mathrm{H}_{2} \mathrm{O}$ features.

\section{3. $\quad H$ band spectrum}

While the $H$ band spectrum falls between the red side of a strong $\mathrm{H}_{2} \mathrm{O}$ band (for $\lambda \lesssim 1.59 \mu \mathrm{m}$ ) and the blue side of a prominent $\mathrm{CH}_{4}$ band (for $\lambda \gtrsim 1.59 \mu \mathrm{m}), \mathrm{NH}_{3}$ and $\mathrm{H}_{2} \mathrm{~S}$ have non-negligible opacity in this wavelength interval which also includes a band of $\mathrm{CO}$.

The strong $\mathrm{CH}_{4}$ band is responsible for the turnover of the flux at $1.59 \mu \mathrm{m}$. In this wavelength range, the $\mathrm{CH}_{4}$ opacity is described in our calculation by the Strong et al. (1993) laboratory measurements which are restricted to $T=300 \mathrm{~K}$. As a consequence, the $\mathrm{CH}_{4}$ band comes in at $1.61 \mu \mathrm{m}$ in the synthetic spectra, which results in strong departures of the mean flux level between data and models for $\lambda>1.58 \mu \mathrm{m}$. This limits our analysis of the $H$ band spectrum to wavelengths shorter than $1.58 \mu \mathrm{m}$. In this wavelength range, 
the spectrum is formed deep in the atmosphere, where $1200<T<1350 \mathrm{~K}$ and $P \sim 10$ bar (Fig. $6)$. Within this spectral region, we determine the abundance of $\mathrm{NH}_{3}$ as a function of the metallicity, but cannot untangle the two. We also comment on the presence of $\mathrm{H}_{2} \mathrm{~S}$ and $\mathrm{CO}$.

\subsubsection{Metallicity and ammonia}

While $\mathrm{NH}_{3}$ absorption can significantly affect the slope of the spectrum shortward of $1.56 \mu \mathrm{m}$ (Fig. 9), there is no distinctive feature at this spectral resolution $(R=2100)$ to provide an unambiguous detection of this molecule. The detection of $\mathrm{CO}$ at $4.7 \mu \mathrm{m}$ well above the equilibrium abundance (Noll et al. 1997) suggests the possibility that $\mathrm{NH}_{3}$ may also depart from its chemical equilibrium abundance (Fegley \& Lodders 1994). We therefore vary the abundance of $\mathrm{NH}_{3}$ by reducing its chemical equilibrium abundance - as computed for a given metallicity - uniformly throughout the atmosphere by constant factor. We found that reduction factors of $1,0.5,0.25$, and 0 provide an adequate grid of $\mathrm{NH}_{3}$ abundances given the $\mathrm{S} / \mathrm{N}$ ratio of the data and the residual problems with the $\mathrm{H}_{2} \mathrm{O}$ opacity.

The effect of varying the metallicity on the $H$ band spectrum is shown in Fig. 10 for model B. All spectra in Figure 10 were computed with the equilibrium abundance of $\mathrm{NH}_{3}$. In this case, we find a best fitting metallicity of $[\mathrm{M} / \mathrm{H}]=-0.3$. Figures 9 and 10 show that varying the abundance of $\mathrm{NH}_{3}$ and varying the metallicity have very similar effects on the synthetic spectrum. In the absence of any distinctive feature of $\mathrm{NH}_{3}$, it is not possible to determine both the metallicity and the $\mathrm{NH}_{3}$ abundance separately. For each value of $[\mathrm{M} / \mathrm{H}]$, we can adjust the $\mathrm{NH}_{3}$ abundance to obtain a good fit, higher metallicities requiring lower $\mathrm{NH}_{3}$ abundances. The best fitting solutions are given in Table 2 . These fits are nearly indistinguishable from each other although the higher metallicity fits are marginally better.

It is in the $H$ band that we find the poorest match in the detailed features of the data and the models. While the fit is determined by matching the relative amplitudes of the features, the twoparameter fit (metallicity and $\mathrm{NH}_{3}$ abundance) we have performed amounts to little more than fitting the slope of the spectrum. As an internal check on our fitting procedure and precision, we have have verified that our fits indeed have the same slope as the data by plotting data and models at a very low spectral resolution which eliminates all absorption features.

\subsubsection{Hydrogen sulfide}

Hydrogen sulfide $\left(\mathrm{H}_{2} \mathrm{~S}\right)$ has non-negligible opacity over most of the wavelength range of our fit to the $H$ band. Figure 11 shows two spectra computed with and without $\mathrm{H}_{2} \mathrm{~S}$ opacity. Its opacity is weaker than that of $\mathrm{NH}_{3}$ however, and there are no distinctive features which would allow a positive identification. Since the $\mathrm{H}_{2} \mathrm{~S}$ features are fairly uniformly distributed in strength and wavelength, fitting the spectrum with either the chemical equilibrium abundance of $\mathrm{H}_{2} \mathrm{~S}$ or with no $\mathrm{H}_{2} \mathrm{~S}$ at all only has a small-to-negligible effect on the determination of the $\mathrm{NH}_{3}$ abundance for a given metallicity (Table 2). For the low gravity model $\mathrm{A}$, the $\mathrm{NH}_{3}$ abundance determined from spectra without $\mathrm{H}_{2} \mathrm{~S}$ is about half of the value found with the chemical equilibrium abundance of $\mathrm{H}_{2} \mathrm{~S}$. The difference decreases at higher gravities and is negligible for model $\mathrm{C}$.

Chemical equilibrium calculations (Fegley \& Lodders 1996; Lodders 1999) indicate that $\mathrm{H}_{2} \mathrm{~S}$ is present in Gl 229B with an abundance essentially equal to the elemental abundance of sulfur in the atmosphere (see $\S 6.3$ for further discussion). Unfortunately, we are unable to ascertain the presence of $\mathrm{H}_{2} \mathrm{~S}$ in the atmosphere of $\mathrm{Gl} 229 \mathrm{~B}$ at present.

\subsubsection{Carbon monoxide}

The discovery of $\mathrm{CO}$ in the $4.7 \mu \mathrm{m}$ spectrum of Gl 229B with an abundance about 3 orders of magnitude higher than predicted by chemical equilibrium revealed the importance of dynamical processes in its atmosphere (Fegley \& Lodders 1996; Noll et al. 1997; Griffith and Yelle 1999). The $4.7 \mu \mathrm{m}$ spectrum probes the atmosphere at the 2 - 3 bar level where $T \sim 900 \mathrm{~K}$ (Fig. 6). At this level, chemical equilibrium calculations predict a CO abundance of $X_{\mathrm{CO}}=4.7 \times 10^{-8}$ while Noll et al. (1997) found $5 \times 10^{-5} \lesssim X_{\mathrm{CO}} \lesssim 2 \times 10^{-4}$. The second overtone band of $\mathrm{CO}$ falls within the $H$ band and, in principle, could provide a determination of $X_{\mathrm{CO}}$ at a deeper level of the atmosphere, where $P \sim 14$ bar and $T \sim 1400 \mathrm{~K}$ (Fig. 
6). Figure 12 shows a comparison of the data with synthetic spectra computed with various amounts of $\mathrm{CO}$ for model B with solar metallicity. The first two spectra are computed with the chemical equilibrium abundance of $\mathrm{CO}\left(X_{\mathrm{CO}}=4.90 \times 10^{-5}\right.$ at 14 bar $)$ and without $\mathrm{CO}\left(X_{\mathrm{CO}}=0\right)$. These two spectra are very nearly identical. A third spectrum is computed in the unrealistic limit where all the carbon in the atmosphere is in the form of $\mathrm{CO}$ $\left(X_{\mathrm{CO}}=2.97 \times 10^{-4}\right)$, which represents the maximum possible $\mathrm{CO}$ enhancement. As we found for $\mathrm{NH}_{3}$ and $\mathrm{H}_{2} \mathrm{~S}$, there is no distinctive spectral signature of $\mathrm{CO}$ at this resolution $(R=2100)$. Our extreme case represents a flux reduction of $\sim 2 \sigma$ at best. We are unable to constrain the $\mathrm{CO}$ abundance with our data. Since the $\Delta \nu=3$ band of $\mathrm{CO}$ is $\sim 10^{3}$ times weaker than the fundamental band at $4.7 \mu \mathrm{m}$, obtaining a useful $\mathrm{CO}$ abundance from $H$ band spectroscopy will be a difficult undertaking.

\section{4. $K$ band spectrum}

Of our three near infrared spectra, the $K$ band spectrum is formed highest in the atmosphere: $T=950-1020 \mathrm{~K}$ and $P \sim 3$ bar (Fig. 6). This is the same level as is probed with $4.7 \mu \mathrm{m}$ spectroscopy. As in the $H$ band, the spectrum contains mainly $\mathrm{H}_{2} \mathrm{O}$ features on the blue side $(\lambda \lesssim 2.1 \mu \mathrm{m})$ and a strong $\mathrm{CH}_{4}$ band appears at longer wavelengths. There are also several $\mathrm{NH}_{3}$ features for $\lambda<2.05 \mu \mathrm{m}$ and the models predict an isolated feature of $\mathrm{H}_{2} \mathrm{~S}$ (Fig. 3).

Figure 13 compares a spectrum computed for model $\mathrm{B}$ with $[\mathrm{M} / \mathrm{H}]=-0.3$ with the entire $K$ band spectrum. There is an excellent agreement in the structure of the spectrum even though the overall shape is not very well reproduced. For $\lambda<2.05 \mu \mathrm{m}$, the model predicts strong features of $\mathrm{NH}_{3}$ which we discuss in the next section. Beyond $2.12 \mu \mathrm{m}$ is a $\mathrm{CH}_{4}$ band which is too weak in the model. The structure within the modeled band is remarkably similar to the observed spectrum, however. This much better agreement of the $\mathrm{CH}_{4}$ band than we obtained in the $H$ band is due to two factors. First, in this band the $\mathrm{CH}_{4}$ opacity is computed from a line list, and can therefore be computed as a function of temperature rather than at a fixed value of $300 \mathrm{~K}$. Second, the lower temperature where the band is formed (Fig. 6) reduces the effect of the incompleteness of the $\mathrm{CH}_{4}$ line list above $300 \mathrm{~K}$. Nevertheless, we do not consider the $\mathrm{CH}_{4}$ features here and limit our analysis to $\lambda \leq 2.10 \mu \mathrm{m}$.

The $K$ band spectrum provides a unique opportunity: Once the metallicity is determined by matching the depth of the $\mathrm{H}_{2} \mathrm{O}$ features between 2.05 and $2.10 \mu \mathrm{m}$, the abundance of $\mathrm{NH}_{3}$ can be obtained by fitting its features below $2.05 \mu \mathrm{m}$. The fit of the metallicity is shown in Fig. 14 for model $\mathrm{B}$, which shows $[\mathrm{M} / \mathrm{H}]=0$ and -0.5 (top panel) and our best fit, $[\mathrm{M} / \mathrm{H}]=-0.3$ (bottom panel). Values for models $\mathrm{A}$ and $\mathrm{C}$ are given in Table 2. All features in this region are due to $\mathrm{H}_{2} \mathrm{O}$ and, as we found in the red spectrum and in the $J$ and $H$ bands, the oscillator strengths of the $\mathrm{H}_{2} \mathrm{O}$ line list do not reproduce the relative strength of the observed features very well.

\subsubsection{Ammonia}

In the wavelength range shown in Fig. 15, the spectrum consists of a few $\mathrm{NH}_{3}$ features on a background of $\mathrm{H}_{2} \mathrm{O}$ absorption. Synthetic spectra predict seven strong $\mathrm{NH}_{3}$ features in this spectrum, three of which are clearly present $(2.033,2.037$ and $2.046 \mu \mathrm{m})$, one is absent $(2.041 \mu \mathrm{m})$ and three appear to be missing $(2.026,2.029$ and $2.031 \mu \mathrm{m})$. This constitutes an ambiguous detection of $\mathrm{NH}_{3}$ in Gl 229B. The determination of the abundance of $\mathrm{NH}_{3}$ from the $K$ band spectrum is hampered by the limited accuracy of the oscillator strengths of the $\mathrm{H}_{2} \mathrm{O}$ line list and by the incompleteness of our $\mathrm{NH}_{3}$ line list for temperatures above $300 \mathrm{~K}$. The effect of the former can be seen in the trio of features at 2.026, 2.029 and $2.031 \mu \mathrm{m}$, which overlap $\mathrm{H}_{2} \mathrm{O}$ absorption features. Even after removing all $\mathrm{NH}_{3}$ opacity, these features are still too strong in the calculated spectrum (top panel of Fig. 15). The top panel of Fig. 15 as well as Fig. 13 show that for model $\mathrm{B}$, the abundance of $\mathrm{NH}_{3}$ derived from the chemical equilibrium for the adopted metallicity of $[\mathrm{M} / \mathrm{H}]=-0.3$ is too high. We therefore consider a depletion in $\mathrm{NH}_{3}$ in the atmosphere of $\mathrm{Gl} 229 \mathrm{~B}$ at the level probed by the $K$ band spectrum. Following the approach used in fitting the $H$ band spectrum, we express this depletion as a fraction of the chemical equilibrium abundance of $\mathrm{NH}_{3}$ for the metallicity obtained independently from the amplitude of the $\mathrm{H}_{2} \mathrm{O}$ features in the $K$ band. This fraction is applied uniformly throughout the atmosphere for the computation 
of the synthetic spectrum. Given the ambiguous presence of $\mathrm{NH}_{3}$ in the $K$ band, we have determined the optimal $\mathrm{NH}_{3}$ abundance by minimizing the $\chi^{2}$ of the spectral fit for $2.022 \leq \lambda \leq 2.049 \mu \mathrm{m}$. This gives a depletion factor of $\lesssim 0.4$ with no $\mathrm{NH}_{3}$ present being an acceptable fit. Restricting the fit to the region where $\mathrm{NH}_{3}$ features are clearly observed $(2.032 \leq \lambda \leq 2.049)$ gives a similar result but favors a finite value for the $\mathrm{NH}_{3}$ depletion of $\sim 0.2$. The results are summarized in Table 2. The lower panel of Figure 15 shows the model B fit obtained by reducing the $\mathrm{NH}_{3}$ abundance throughout the atmosphere to $\sim 25 \%$ of its chemical equilibrium value for the adopted metallicity. With this significant degree of depletion, the model reproduces the three detected features $(2.033,2.037$ and $2.046 \mu \mathrm{m})$ extremely well and makes the $2.041 \mu \mathrm{m}$ feature consistent with the observations.

Because our $\mathrm{NH}_{3}$ line list is incomplete at high temperatures, the opacity which we compute is strictly a lower limit to the actual $\mathrm{NH}_{3}$ opacity at any wavelength. If the incompleteness is significant for the features found in the $K$ band spectrum, then the $\mathrm{NH}_{3}$ abundance is actually lower still. It appears extremely unlikely that the errors in the oscillator strength of the $\mathrm{H}_{2} \mathrm{O}$ transitions would conspire to mimic the depletion of $\mathrm{NH}_{3}$ which we find. For example, if we imagine that there is no $\mathrm{NH}_{3}$ depletion (dotted curve in the top panel of Fig. 14), the residuals between the data and the fitted spectrum for $\lambda<2.05 \mu \mathrm{m}$ would be much larger than the typical mismatch which we find in $\mathrm{H}_{2} \mathrm{O}$ features in all four spectra presented here. We consider the depletion of $\mathrm{NH}_{3}$ in the $K$ band spectrum to be firmly established.

\subsubsection{Hydrogen sulfide}

We are not able to establish the presence of $\mathrm{H}_{2} \mathrm{~S}$ from our $H$ band spectrum (§5.3.2). Throughout the $K$ band, the $\mathrm{H}_{2} \mathrm{~S}$ opacity is generally overwhelmed by $\mathrm{H}_{2} \mathrm{O}$ and $\mathrm{CH}_{4}$ absorption. However, there is a peak in the opacity of $\mathrm{H}_{2} \mathrm{~S}$ which is about one order of magnitude higher than all other opacity maxima (Fig. 16). Our synthetic spectra indicate that this feature is strong enough to become visible in the midst of the background of $\mathrm{H}_{2} \mathrm{O}$ and $\mathrm{CH}_{4}$ features. Figure 17 shows the relevant portion of the $K$ band spectrum with synthetic spectra for all three models (A, B, and C) using the fitted metallicity (Table 2). All three panels are remarkably similar. The strength of the predicted feature is well above the noise level of the data, and taken at face value, Figure 17 indicates a probable depletion of $\mathrm{H}_{2} \mathrm{~S}$ by more than a factor of 2 . On the other hand, chemical equilibrium calculations indicate that the $\mathrm{H}_{2} \mathrm{~S}$ abundance should be very near the elemental abundance of sulfur, even in the presence of vertical transport and condensation (Fegley \& Lodders 1996). Since there is no reason to expect a significant depletion of $\mathrm{H}_{2} \mathrm{~S}$, the discrepancy is probably due to remaining uncertainties in the opacities. Our $\mathrm{H}_{2} \mathrm{~S}$ line list is based on an ab initio calculation (R. Wattson, priv. comm.) which hasn't been compared to laboratory data in this part of the spectrum. The strong feature centered at $2.1084 \mu \mathrm{m}$ is a blend of three strong lines from three different bands of $\mathrm{H}_{2} \mathrm{~S}$. Possible errors in the position or strength of these lines could significantly reduce the amplitude of the feature in our synthetic spectra. The limitations of the background opacity of $\mathrm{H}_{2} \mathrm{O}$ and $\mathrm{CH}_{4}$ may also be responsible for the observed mismatch. Nevertheless, it is desirable to look for this feature at a higher resolution and a higher $\mathrm{S} / \mathrm{N}$ ratio as an absence of sulfur in Gl 229B would be a most intriguing result.

\subsection{Carbon monoxide at $4.7 \mu \mathrm{m}$}

Given our determination of $T_{\text {eff }}$ and $[\mathrm{M} / \mathrm{H}]$ in terms of the surface gravity, we can obtain the abundance of $\mathrm{CO}$ from the $4-5 \mu \mathrm{m}$ spectra of Noll et al. (1997) and Oppenheimer et al. (1998) which is consistent with our results. For each model - A, $\mathrm{B}$, and $\mathrm{C}$ - and using the metallicity given in Table 2 , we computed synthetic spectra with various $\mathrm{CO}$ abundances. The latter is varied freely without imposing stoichiometric constraints. The synthetic spectra are binned to the wavelength grid of the data and fitted to the data by a normalization factor adjusted to minimize the $\chi^{2}$. The $\chi^{2}$ of the fitted spectra shows a well-defined minimum as a function of the $\mathrm{CO}$ abundance, $X_{\mathrm{CO}}$ (Table 3). The uncertainty on the $\mathrm{CO}$ abundance is obtained by generating synthetic data sets by adding a gaussian distribution of the observed noise to the best fitting model spectrum. After doing the same analysis on 1000 synthetic data sets, we obtain a (non-Gaussian) distribution of values of $X_{\mathrm{CO}}$. The uncertainties given in Table 3 
correspond to the $68 \%$ confidence level. The Oppenheimer et al. (1998) spectrum gives CO abundances which are 0.1 dex higher than those obtained from the Noll et al. (1997) spectrum; which is well within the fitting uncertainty. Noll et al. (1997) found a CO mole fraction of 50 to $200 \mathrm{ppm}$ $\left(-4.3 \leq \log X_{\mathrm{CO}} \leq-3.7\right)$ by assuming a $\mathrm{H}_{2} \mathrm{O}$ abundance of $300 \mathrm{ppm}$ (effectively, $[\mathrm{M} / \mathrm{H}]=-0.6$ ), which agrees well with our result for model A which has $[\mathrm{M} / \mathrm{H}]=-0.5$. Our results are also consistent with those of Griffith and Yelle (1999) who find $\mathrm{CO}$ abundances of $\geq 20$ and $\geq 100 \mathrm{ppm}$ $\left(\log X_{\mathrm{CO}} \geq-4.7\right.$ and -4$)$ for $[\mathrm{M} / \mathrm{H}]=-0.6$ and 0 , respectively, using the data of Noll et al. (1997).

Since this part of the spectrum contains only $\mathrm{H}_{2} \mathrm{O}$ and $\mathrm{CO}$ features, our fitting procedure is sensitive only to the $\mathrm{CO}$ to $\mathrm{H}_{2} \mathrm{O}$ abundance ratio. Chemical equilibrium calculations show that the $\mathrm{H}_{2} \mathrm{O}$ abundance scales linearly with the metallicity at the level probed with $4-5 \mu \mathrm{m}$ spectroscopy (i.e. all the gas phase oxygen is in $\mathrm{H}_{2} \mathrm{O}$ ). Accordingly, the $\mathrm{CO}$ abundance we find scales with the metallicity of the model. As shown in Figure 18, the $\mathrm{CO}$ abundance determined from the $4.7 \mu \mathrm{m}$ band corresponds approximately to the $\mathrm{CO} / \mathrm{CH}_{4}$ transition in chemical equilibrium. The observations definitely exclude the very-low chemical equilibrium abundance of $\mathrm{CO}$. The fact that the extreme case where all carbon is $\mathrm{CO}$ provides an acceptable fit to the data (while we know that a good fraction of the carbon is in $\mathrm{CH}_{4}$ ) is due to the rather noisy spectra.

\section{Discussion}

\subsection{Surface Gravity}

It is highly desirable to restrict the surface gravity $g$ of Gl 229B to an astrophysically useful range. Since $L_{\mathrm{bol}}$ is known, a determination of $g$ fixes $T_{\mathrm{eff}}$, the radius, the mass, and the age of Gl 229B (Fig. 4 ), as well as the metallicity and the abundances of important molecules such as $\mathrm{CO}$ and $\mathrm{NH}_{3}$. The large uncertainty on $g$ results in a large uncertainty in the mass of Gl 229B and on the age of the system determined from cooling tracks. While a dynamical determination of the mass may be possible in a decade or so (Golimowski et al. 1998), a spectroscopic determination might be obtained much sooner.

Unfortunately, it is not possible to constrain the gravity better than $\log g=5 \pm 0.5$ with the data and models presently available. Our high resolution spectroscopy does not allow us to choose between models A, B and C (Table 2) as an increase in gravity can be compensated by an increase in metallicity to lead to an identical fit. The gravity sensitivity of the $K$ band synthetic spectrum models reported by Burrows et al. (1999a) occurs for a fixed metallicity only.

Similarly, Allard et al. (1996) and Burrows et al. (1999a) report that the spectral energy distribution of Gl 229B models is fairly sensitive to the gravity. But this is true only for a fixed metallicity. For the three models indicated in Fig. 4, and using the metallicity we have determined for each (Table 2) the gravity dependence of the infrared colors is $\Delta(H-K) / \Delta(\log g)=0.03$ and $\Delta(J-$ $K) / \Delta(\log g)=0.03$ and $\Delta\left(K-L^{\prime}\right) / \Delta(\log g)=$ -0.08 (Table 4$)$. This dependence is very weak in the light of the uncertainty in the photometry of Gl 229B. More problematic is the fact that the synthetic $H-K$ disagrees with the photometry. Furthermore, the incomplete $\mathrm{CH}_{4}$ opacities used in the spectrum calculation almost certainly result in an inaccurate redistribution of the flux in the near infrared opacity windows which determines the broad band colors. An example of this effect on the $H$ band flux can be seen in Fig. 10. We conclude that a photometric determination of the gravity is not possible at present.

An alternative approach to determining the gravity of Gl $229 \mathrm{~B}$ is through a study of the pressure-broadened molecular lines of its spectrum. The spectrum of $\mathrm{Gl} 229 \mathrm{~B}$ is formed of a forest of unresolved molecular lines maily due to $\mathrm{H}_{2} \mathrm{O}, \mathrm{CH}_{4}$, and $\mathrm{NH}_{3}$. Because of the limitations of the $\mathrm{CH}_{4}$ and $\mathrm{NH}_{3}$ opacity data bases, a detailed study of molecular features is best performed in spectral domains where these two molecules do not contribute significantly to the opacity. Spectroscopic observations with a resolution of $\sim 50000$ can reveal the shape of individual $\mathrm{H}_{2} \mathrm{O}$ lines in regions where they are relatively sparse, e.g. from 2.08 to $2.105 \mu \mathrm{m}$.

\subsection{Metallicity}

Our determination of the metallicity of Gl $229 \mathrm{~B}$, with an uncertainty of \pm 0.1 , is given in Table 2 for the three gravities considered. We find an excellent agreement between our three inde- 
pendent determinations of $[\mathrm{M} / \mathrm{H}]$ for each gravity and conclude that Gl 229B is likely to be depleted in heavy elements, e.g. oxygen. The metallicity is near solar at high gravity and decreases significantly for lower gravities. In their analysis of the "red" spectrum, Griffith et al. (1998) found a $\mathrm{H}_{2} \mathrm{O}$ abundance between 0.3 and 0.45 of the solar value for a $T_{\text {eff }}=900 \mathrm{~K}, \log g=5$ model and they adopt a value of 0.25 in Griffith and Yelle (1999).

Metal depletion in Gl 229B is consistent with the analysis of the $0.985-1.02 \mu \mathrm{m} \mathrm{FeH}$ band in the spectrum of the primary star Gl 229A by Schiavon, Barbuy \& Singh (1997) who find $[\mathrm{Fe} / \mathrm{H}]=-0.2$. The infrared colors of Gl 229A imply that it is slightly metal-rich, however (Leggett 1992). The relative metallicity of the components of this binary system may have been affected by their formation process. If the pair formed from the fragmentation of a collapsing cloud (like a binary star system), the two objects should share the same composition. If the brown dwarf formed like a planet, from accretion within a dissipative Keplerian disk around the primary, the selective accretion of solid phase material could lead to an enrichment in heavy elements compared to the primary star, as is observed in the gaseous planets of the solar system. The low mass of the primary $\left(\sim 0.5 M_{\odot}\right)$ and the large semi-major axis and eccentricity $(a \gtrsim 32 \mathrm{AU}$ and $e \gtrsim 0.25$, Golimowski et al. (1998)) suggests that the binary formation process, and therefore equal metallicities, are more plausible. A more detailed study of the metallicity of Gl 229A is desirable to better understand the history of this system.

\subsection{Atmospheric chemistry and molecu- lar abundances}

The results of our analysis of the metallicity and abundances of several molecules in the atmosphere of Gl 229B are summarized in Fig. 18. Each panel corresponds to a different model (see Table 2) and shows the abundances of important molecules as a function of depth in the atmosphere based on chemical equilibrium calculations including condensation cloud formation. The chemistry of these abundant molecules is fairly simple. The abundance of $\mathrm{H}_{2} \mathrm{O}$ is uniformly reduced by $\sim 16 \%$ by silicate condensation. Except for a small depletion for $\log T \lesssim 3$ due to the condensation of $\mathrm{Na}_{2} \mathrm{~S}$, all sulfur is found in $\mathrm{H}_{2} \mathrm{~S}$. The other molecules shown are not affected by condensation. Nitrogen is partitioned between $\mathrm{N}_{2}$ and $\mathrm{NH}_{3}$, with the latter being favored at lower temperatures and higher pressures. $\mathrm{NH}_{3}$ dominates near the surface and rapidly transforms into $\mathrm{N}_{2}$ at the higher temperatures found deeper in the atmosphere. Deep in the atmosphere, the higher pressures cause a partial recombination of $\mathrm{NH}_{3}$ and the ratio of the $\mathrm{NH}_{3}$ to $\mathrm{N}_{2}$ abundances increases slowly with depth. In a similar fashion, all elemental carbon is found in $\mathrm{CH}_{4}$ at the surface but $\mathrm{CO}$ starts to form at higher temperatures and rapidly becomes the most abundant carbon-bearing molecule. The formation of CO consumes $\mathrm{H}_{2} \mathrm{O}$, as can be seen in Fig. 18 .

In each panel, a dotted box indicates the $\mathrm{CO}$ abundance we have determined from the $4.7 \mu \mathrm{m}$ spectrum, the location of the box along the ordinate shows the level probed at this wavelength (Fig. 6). As originally discussed by Noll et al. (1997) and Griffith and Yelle (1999), the CO abundance is $\sim 3$ orders of magnitude larger than the chemical equilibrium value. Stochiometric constraints imply that this also results in a significant reduction of the $\mathrm{CH}_{4}$ abundance at the 870 - $950 \mathrm{~K}$ level in Gl 229B. In model B, the equilibrium abundance of $\mathrm{CH}_{4}$ at $900 \mathrm{~K}$ is $2.97 \times 10^{-4}$, which corresponds to the abundance of elemental carbon. The $\mathrm{CO}$ abundance determined from the $4.7 \mu \mathrm{m}$ band is $1.6 \times 10^{-4}$ (with a large error bar). Conservation of the total number of carbon atoms then requires that the $\mathrm{CH}_{4}$ abundance be $\sim 1.4 \times 10^{-4}$, a full factor of 2 below the equilibrium abundance. If we use the lowest $\mathrm{CO}$ abundance allowed by our analysis, a $25 \%$ reduction of $\mathrm{CH}_{4}$ relative to its equilibrium abundance at $900 \mathrm{~K}$ results. Depletion of $\mathrm{CH}_{4}$ at this depth is readily accessible spectroscopically in the 1.6 and $3.3 \mu \mathrm{m}$ bands, and may also affect the $2.3 \mu \mathrm{m}$ band if the non-equilibrium $\mathrm{CO}$ abundance persists at higher levels (Fig. 6). After $\mathrm{H}_{2} \mathrm{O}, \mathrm{CH}_{4}$ is the most important near infrared molecular absorber in Gl 229B. Accurate modeling of the spectrum demands a careful treatment of the non-equilibrium $\mathrm{CH}_{4}$ abundance.

Similarly, solid boxes show the $\mathrm{NH}_{3}$ abundance determined from our $H$ and $K$ band spectra. While the $H$ band abundance is in excellent agreement with the equilibrium value, there is a clear depletion of $\mathrm{NH}_{3}$ in the $K$ band. The $H$ and $K$ band abundances are marginally consistent with 
each other but it appears that the $\mathrm{NH}_{3}$ abundance decreases upwards through the atmosphere.

\subsection{Non-equilibrium processes}

Processes which take place faster than the time scale of key chemical reactions can drive the composition of the mixture away from equilibrium. The case of $\mathrm{CO} / \mathrm{CH}_{4}$ chemistry has been wellstudied in the atmosphere of Jupiter where an overabundance of $\mathrm{CO}$ is also observed. Carbon monoxyde is a strongly bound molecule and the conversion of $\mathrm{CO}$ to $\mathrm{CH}_{4}$ through the (schematic) reaction

$$
\mathrm{CO}+3 \mathrm{H}_{2} \rightarrow \mathrm{CH}_{4}+\mathrm{H}_{2} \mathrm{O}
$$

proceeds relatively slowly, while the reverse reaction is much faster. Vertical transport, if vigorous enough, can carry CO-rich gas from deeper levels upwards faster than the $\mathrm{CO}$ to $\mathrm{CH}_{4}$ reaction can take place. The $\mathrm{CO} / \mathrm{CH}_{4}$ ratio at any level is fixed ("quenched") by the condition

$$
\tau_{\text {chem }}=\tau_{\text {mix }},
$$

where $\tau_{\text {chem }}$ is the chemical reaction time scale and $\tau_{\text {mix }}$ the dynamical transport time scale. Wherever $\tau_{\text {mix }} \leq \tau_{\text {chem }}$ in the presence of a vertical gradient in the equilibrium abundance, nonequilibrium abundances will result.

As discussed by Fegley \& Lodders (1996), Noll et al. (1997), Griffith and Yelle (1999) (and references therein), this naturally explains the very high CO abundance observed at the $900 \mathrm{~K}$ level. In this picture, CO-rich gas would be carried upward from $T \gtrsim 1400 \mathrm{~K}$. Convection is the most obvious form of vertical transport in a stellar atmosphere but in Gl 229B the convection zone remains about 3 pressure scale heights below the level where $\mathrm{CO}$ is observed (shaded area in Fig. 18). Perhaps convective overshooting can transport $\mathrm{CO}$ to the observed level. Griffith and Yelle (1999) propose "eddy diffusion" as a slower, yet adequate transport mechanism. The eddy diffusion (or mixing) time scale is constrained by the poorly known $\mathrm{CO}$ abundance and the somewhat uncertain chemical pathway between $\mathrm{CO}$ and $\mathrm{CH}_{4}$. From Griffith and Yelle (1999), we infer that $\tau_{\text {mix }} \lesssim 1$ to 10 years and could be much smaller.

In analogy to the $\mathrm{CO} / \mathrm{CH}_{4}$ equilibrium, a low $\mathrm{NH}_{3}$ abundance can be explained by vertical transport which can quench the $\mathrm{NH}_{3} / \mathrm{N}_{2}$ ratio at a value found in deeper layers in the atmosphere. As it is carried upward, $\mathrm{N}_{2}$ is converted to $\mathrm{NH}_{3}$ by the reaction

$$
\mathrm{N}_{2}+3 \mathrm{H}_{2} \rightarrow 2 \mathrm{NH}_{3}
$$

The $\mathrm{N}_{2}$ molecule is very strongly bound, however, and this reaction proceeds extremely slowly at low temperatures, much more slowly than reaction (2). Thermochemical kinetic calculations of the chemical lifetime for conversion of $\mathrm{N}_{2}$ to $\mathrm{NH}_{3}$ were performed as described in Fegley \& Lodders (1994). The time scale for reaction (4) along the $(P, T)$ profiles of models A, B, and $\mathrm{C}$ and for $P>1$ bar is given by

$$
\log \tau_{\text {chem }}=\frac{3.75 \times 10^{4}}{T}+0.22 \log g-23.08,
$$

where $\tau_{\text {chem }}$ is in year, $T$ is the temperature in $\mathrm{K}$, and $g$ is the surface gravity in $\mathrm{cm} / \mathrm{s}^{2}$. This time scale assumes that the $\mathrm{N}_{2}$ conversion occurs in the gas phase although it could possibly be shortened by catalysis on the surface of grains. The time scale increases very steeply with decreasing temperature. At the level probed in the $H$ band, $\log T=3.1$ and $\log \tau_{\text {chem }}=7.8$ and in the $K$ band, $\log T=2.95$ and $\log \tau_{\text {chem }}=20.1$ ! At some intermediate level (which depends on $g$ ), the time scale for the conversion of $\mathrm{N}_{2}$ into $\mathrm{NH}_{3}$ becomes longer than the age of Gl 229B. In view of the relatively very short mixing time scale inferred from the $\mathrm{CO}$ abundance, it follows that the $\mathrm{NH}_{3}$ abundance in the $H$ and $K$ bands is entirely determined by non-equilibrium processes, not by reaction (4). At depths where $\log T>3.23$ (which corresponds to the top of the convection zone), $\tau_{\text {chem }}<1 \mathrm{yr}$ and the reaction proceeds fast enough to establish chemical equilibrium between $\mathrm{NH}_{3}$ and $\mathrm{N}_{2}$. We therefore expect that the $\mathrm{N}_{2} / \mathrm{NH}_{3}$ ratio will be quenched at its value at $\log T \approx 3.23$, throughout the remainder of the atmosphere. While the time scale for eddy diffusion increases in the upper levels of the atmosphere, the extremely long $\tau_{\text {chem }}$ ensures that the $\mathrm{NH}_{3} / \mathrm{N}_{2}$ ratio remains unchanged, regardless of how slowly the vertical mixing proceeds. For model $\mathrm{B}$, this corresponds to $\log X_{\mathrm{NH}_{3}}=-5.2$, in perfect agreement with the abundance found in the $H$ band (Fig. 18b).

While the abundance of $\mathrm{NH}_{3}$ determined from the $K$ band spectrum is not very precise, it is 
marginally consistent with vertical mixing. Figure 18 shows that it is more likely that the $K$ band abundance is smaller than found in the $H$ band, however. On the other hand, the abundances shown in Fig. 18 and Table 2 are depletion factors which were applied uniformly to the chemical equilibrium abundance profile of $\mathrm{NH}_{3}$, which has a large vertical gradient. For consistency with the mixed atmosphere picture, we have therefore redetermined $\mathrm{NH}_{3}$ abundances using a constant abundance throughout the atmosphere and found $\log X_{\mathrm{NH}_{3}} \leq-5.0$ and $-4.7 \pm 0.15$ from the $K$ and $H$ band spectra, respectively (model B). The former is in good agreement with our simple prediction while the $H$ band value is now rather high. The discrepancy between the $H$ and $K$ band results thus persists in this new analysis. Changes in $[\mathrm{M} / \mathrm{H}]$ within the \pm 0.1 uncertainty have little effect either.

We consider the possibility that this vertical gradient in the $\mathrm{NH}_{3}$ abundance may be caused by a different non-equilibrium process such as the photolysis of $\mathrm{NH}_{3}$ by the UV flux from the primary star. Ammonia is a relatively fragile molecule which is easily dissociated by UV photons:

$$
\mathrm{NH}_{3}+h \nu \rightarrow \mathrm{NH}_{2}+\mathrm{H} .
$$

With a photodissociation cross section of $\approx 6 \times$ $10^{-18} \mathrm{~cm}^{2}$ per molecule, optical depth unity for the photodissociation of $\mathrm{NH}_{3}$ is reached at pressures of a few millibars in Gl 229B. Photodissociation of the much more abundant $\mathrm{H}_{2}$ molecules does not effectively shield $\mathrm{NH}_{3}$ from incoming UV photons since the two molecules absorb over different wavelength ranges. Photodissociation of $\mathrm{NH}_{3}$ therefore represents a net sink of $\mathrm{NH}_{3}$ which occurs at the very top of the atmosphere. We can estimate a lower limit to the time scale of photodissociation of $\mathrm{NH}_{3}$ by assuming that each photodissociating photon results in the destruction of a $\mathrm{NH}_{3}$ molecule. The incident photon flux is

$$
N_{\mathrm{UV}}=\left(\frac{R_{\star}}{d}\right)^{2} \int_{\lambda_{0}}^{\lambda_{1}} \frac{\lambda \mathcal{F}_{\lambda}}{h c} d \lambda \approx\left(\frac{R_{\star}}{d}\right)^{2} \frac{\bar{\lambda} \mathcal{F}_{\bar{\lambda}}}{h c} \Delta \lambda,
$$

where photons causing dissociation of $\mathrm{NH}_{3}$ are between $\lambda_{0}$ and $\lambda_{1}, \mathcal{F}_{\lambda}$ is the flux at the surface of the primary star, $R_{\star}$ the radius of the primary star and $d$ the separation of the binary system. For $\mathrm{NH}_{3}$, we have $\bar{\lambda} \sim 1900 \AA$ and $\Delta \lambda \sim 300 \AA$ (Moses, priv. comm.). The primary star has a dM1 spectral type, with $\mathcal{F}_{\bar{\lambda}} \sim 10^{7} \mathrm{erg} \mathrm{cm}^{-2} \mathrm{~s}^{-1} \mathrm{~cm}^{-1}$ and $R_{\star} \sim 3.6 \times 10^{10} \mathrm{~cm}$ (Leggett et al. 1996). The binary separation is $d \gtrsim 44 \mathrm{AU}$ (Golimowski et al. 1998). This results in $N_{\mathrm{UV}} \lesssim 8.3 \times 10^{3} \mathrm{~cm}^{-2} \mathrm{~s}^{-1}$. Photodissociation will affect significantly the $\mathrm{NH}_{3}$ abundance when

$$
\tau_{\text {phot }}=\frac{\sigma}{N_{\mathrm{UV}}} \lesssim \tau_{\text {mix }},
$$

where $\sigma$ is the column density of $\mathrm{NH}_{3}$. Because the incident flux of UV photons is fairly low, this condition is satisfied only at pressures below a few microbars, i.e. very high in the atmosphere. In the region of interest, photodissociation destroys a very small fraction of $\mathrm{NH}_{3}$ during one mixing time scale and therefore has little effect on the abundance of $\mathrm{NH}_{3}$. Photolysis of $\mathrm{NH}_{3}$ cannot explain the relatively low $\mathrm{NH}_{3}$ abundance we find in the $K$ band spectrum.

We believe that the difference between the $H$ and $K$ band determinations of the $\mathrm{NH}_{3}$ abundance arise from the limitations of the $\mathrm{NH}_{3}$ opacity data used for the calculation of the synthetic spectra. As discussed above, the incompleteness of the $\mathrm{NH}_{3}$ line list for $T>300 \mathrm{~K}$ results in upper limits for the $\mathrm{NH}_{3}$ abundances obtained by fitting the data. Since this effect increases with temperature, we expect that the abundance determined from the $H$ band spectrum is overestimated relative to the one obtained from the $K$ band spectrum, which is what we observe. Until $\mathrm{NH}_{3}$ opacities become available for $T \sim 800-1200 \mathrm{~K}$, we will not be able to quantify this effect.

Figures 6 and 18 show that ammonia offers a third window of opportunity for a determination of its abundance. The region between 8.3 and $14.4 \mu \mathrm{m}$ is rich in strong $\mathrm{NH}_{3}$ features, the two strongest being at 10.35 and $10.75 \mu \mathrm{m}$ (Fig. 19). This spectral region probes a higher level in the atmosphere $(T \sim 500-800 \mathrm{~K})$ where the hot bands of $\mathrm{NH}_{3}$ which are missing from opacity data bases are less problematic than in the $H$ and $K$ bands. In this spectral region, the spectrum is very sensitive to the $\mathrm{NH}_{3} / \mathrm{H}_{2} \mathrm{O}$ ratio, especially for $\mathrm{NH}_{3}$ abundances below $25 \%$ of the equilibrium value (Fig. 19). This would allow a good determination of the degree of $\mathrm{NH}_{3}$ depletion in the upper levels of the atmosphere. We anticipate that $10 \mu \mathrm{m}$ spectroscopy should reveal a $\mathrm{NH}_{3}$ abundance of $\lesssim 10 \%$ of its equilibrium value. 


\section{Conclusion}

With the availability of extensive photometric, astrometric, and spectroscopic data, our picture of the atmosphere of Gl 229B is gradually becoming more exotic and more complex. The initial discovery of $\mathrm{CH}_{4}$ in its spectrum set it appart and has prompted the creation of a new spectral class, the T dwarfs. $\mathrm{H}_{2} \mathrm{O}, \mathrm{CO}, \mathrm{Cs} \mathrm{I}$, and $\mathrm{K} \mathrm{I}$ have also been detected. There is good evidence that the rapid decrease of the flux at visible wavelengths is caused by unprecedently broad lines of atomic alkali metals (Liebert et al. 2000). The presence of condensates may also play a role in shaping the spectrum of Gl 229B.

It is unfortunate that the surface gravity of Gl 229B remains poorly constrained. We have not been able to further restrict the allowed range with our new $J, H$, and $K$ spectroscopy. As a result, all our results are expressed as a function of gravity. This is the most significant obstacle to further progress in elucidating the astrophysics of this $\mathrm{T}$ dwarf. The surface gravity can probably be determined from the study of the pressure broadened shape of molecular lines.

We have found good evidence for the presence of $\mathrm{NH}_{3}$ in the spectrum of $\mathrm{Gl} 229 \mathrm{~B}$, which was expected from chemical equilibrium calculations. We have been able to determine its abundance at two different levels in the atmosphere, and we find a significant deviation from chemical equilibrium. A similar situation has been found with CO previously (Noll et al. 1997) and this abundance pattern can be explained by vertical mixing in the atmosphere. The extent of the convection zone is not sufficient to account for the abundances we find and the mixing may be due to overshooting or to less efficient eddy diffusion. We find that $\mathrm{NH}_{3}$ photolysis is not important in shaping the spectrum of Gl 229B. Because $\mathrm{NH}_{3}$ can be observed in three different bands corresponding to three distinct depths in the atmosphere, an accurate determination of its abundance in each band provides information on the time scale of mixing as a function of depth. This is an unusual and powerful diagnostic tool which can provide valuable clues for modeling the vertical distributions of possible condensates. In principle, any absorber with a large abundance gradient through the visible part of the atmosphere can be used to infer the details of the mixing process. Among detected and abundant molecules, only $\mathrm{CO}$ and $\mathrm{NH}_{3}$ satisfy this criterion. Chemical equilibrium calculations with rainout of condensates (Lodders 1999; Burrows et al. 1999b) show that we can expect significant vertical gradients in the abundances of atomic $\mathrm{K}, \mathrm{Rb}$, $\mathrm{Cs}$, and $\mathrm{Na}$ as they become bound in molecules ( $\mathrm{KCl}, \mathrm{RbCl}, \mathrm{CsCl}$ and $\mathrm{Na}_{2} \mathrm{~S}$, respectively) in the cooler, upper reaches of the atmosphere. Cesium and potassium have been detected in the spectrum of Gl 229B, and resonance doublets of K I and $\mathrm{Na}$ I appear to shape the visible spectrum. However, the chemical timescales for alkali metals are so short that they should always remain in thermodynamic equilibrium (Lodders 1999). Therefore, they cannot serve as probes of vertical mixing in Gl 229B.

Further progress in understanding the atmosphere of Gl 229B requires better opacities for $\mathrm{CH}_{4}$ and $\mathrm{NH}_{3}$, and, to a lesser extent, of $\mathrm{H}_{2} \mathrm{O}$. A more accurate determination of the $\mathrm{CO}$ abundance from $4-5 \mu \mathrm{m}$ spectroscopy is very desirable and will require higher signal-to-noise spectroscopy than is currently available. Similarly, $10 \mu \mathrm{m}$ spectroscopy to determine the $\mathrm{NH}_{3}$ abundance for $P \lesssim 1$ bar, while difficult, is important.

The issue of vertical mixing and departures from chemical equilibrium gains importance when we consider that the observed departure of $\mathrm{CO}$ from chemical equilibrium implies a significantly reduced $\mathrm{CH}_{4}$ abundance, by conservation of the abundance of elemental carbon. Similarly, our results imply that $\mathrm{NH}_{3}$ absorption in the $10 \mu \mathrm{m}$ region is reduced. Because $\mathrm{CH}_{4}$ is a significant absorber in the near infrared, as is $\mathrm{NH}_{3}$ in the $10 \mu \mathrm{m}$ range, departures from equilibrium must be taken into account when accurate modeling of the atmosphere and spectrum of Gl 229B is desired. This new level of complexity compounds the exoticism and the challenges posed by $\mathrm{T}$ dwarfs.

The astrophysics of Gl 229B is far richer than has been originally anticipated. Gl $229 \mathrm{~B}$ is currently the only $\mathrm{T}$ dwarf known to be in a binary system. There is no evidence that the illumination from the primary star has a significant effect on the state of its atmosphere and Gl 229B is most likely typical of isolated $\mathrm{T}$ dwarfs. It remains the brightest and by far the best studied of the seven T dwarfs currently known, but the list should expand to several dozens during the 
next $2-3$ years (Burgasser et al. 1999; Strauss et al. 1999). The existing body of work on Gl $229 \mathrm{~B}$ points to the most rewarding observations to conduct on $\mathrm{T}$ dwarfs. The possibility of studying trends in the physics of $\mathrm{T}$ dwarf atmospheres as a function of effective temperature is a fascinating prospect.

We thank T. Guillot for sharing programs which were most useful to our analysis, J. Moses for invaluable information regarding the photolysis of $\mathrm{NH}_{3}$ in giant planets, and K. Noll and B. Oppenheimer for sharing their data. We are grateful to the staff at the United Kingdom Infrared Telescope, which is operated by the Joint Astronomy Center Hawaii on behalf of the UK Particle Physics and Astronomy Research Council. This work was supported in part by NSF grants AST-9318970 and AST-962487 and NASA grants NAG5-4988 and NAG5-4970. Work by B. Fegley and K. Lodders is supported by grant NAG5-6366 from the NASA Planetary Atmospheres Program.

\section{REFERENCES}

Allard, F., Hauschildt, P.H., Baraffe, I. \& Chabrier, G. 1996, ApJ, 465, L123

Allard, F. 1998, in Brown Dwarfs and Extrasolar Planets, ASP. Conf. Series Vol 134, Rebolo, R., Martin, E.L. and Zapatero Osorio, M.R., Eds., p370

Allard, F., Alexander, D.R., Tamanai, A. \& Hauschildt, P.H. 1998, in Brown Dwarfs and Extrasolar Planets, ASP. Conf. Series Vol 134, Rebolo, R., Martin, E.L. and Zapatero Osorio, M.R., Eds., p438.

Anders, E., \& Grevesse, N. 1989, Geo. Chem. Acta., 53, 197

Bohren, C.F. \& Huffman, D.R. 1983, Absorption and Scattering of Light by Small Particles, (Wiley: New York)

Burgasser, A.J., Kirkpatrick, J.D., Brown, M.E., Reid, I.N., Gizis, J.E., Dahn, C.C., Monet, D.G., Beichman, C.A., Liebert, J., Cutri, R.M., \& Skrutskie, M.F. 1999, ApJ, 552, L65

Burrows, A., Marley, M.S., Hubbard, W.B., Lunine, J.I., Guillot, T., Saumon, D., Freedman, R.S., Sudarsky, D., \& Sharp, C. 1997, ApJ, 491, 856

Burrows, A., Hubbard, W.B., Lunine, J.I., Marley, M.S., \& Saumon, D. 1999a, in Protostars and Planets IV, V., Mannings, A. Boss, S. Russell, eds., (University of Arizona Press: Tucson) in press.

Burrows, A., Marley, M.S., \& Sharp, C. 1999b, submitted to ApJ, astro-ph9908078

Burrows, A., \& Sharp, C.M. 1999, ApJ, 512, 843

Fegley, B. Jr., \& Lodders, K. 1994, Icarus, 110, 117

Fegley, B. Jr., \& Lodders, K. 1996, ApJ, 472, L37

Fegley, B. Jr., \& Prinn, R.G. 1988, ApJ, 324, 621

Geballe, T.R., Kulkarni, S.R., Woodward, C.E. \& Sloan, G.C. 1996, ApJ, 467, L101

Golimowski, D.A., Burrows, C.J., Kulkarni, S.R., Oppenheimer, B.R., \& Brukardt, R.A. 1998, AJ, 115,2579 
Grevesse, N., \& Noels, A. 1993, in Origin and Evolution of the Elements, Prantzos et al., eds. (Cambridge University Press: Cambridge), p15.

Griffith, C.A., Yelle, R.A. \& Marley, M.S. 1998, Science, 282, 2063

Griffith, C.A. \& Yelle, R.A. 1999, ApJ, 519, L85

Guillot, T. 1999, Science, 286, 72

Guillot, T., Saumon, D., Burrows, A., Hubbard, W.B., Lunine, J.I., Marley, M.S., \& Freedman, R.S. 1996, in "Astronomical and Biochemical Origins and the Search for Life in the Universe," Eds. C.B. Cosmovici, S. Bowyer and D. Werthimer, Editrice Compositori, 343

Husson, N., Bonnet, B., Scott, N.A., \& Chedin, A. 1994, J. Quant. Spec. Radiat. Transf., 48, 509

Hubbard, W.B., Burrows, A., Lunine, J.I., \& Saumon, D. 1995, in Elementary Processes in Dense Plasmas, S. Ichimaru, and S. Ogata, Eds., (Addison-Wesley: Reading), p227

Jones, H.R.A. \& Tsuji, T. 1997, ApJ, 480, L39

Karkoschka, E. 1994, Icarus, 111, 174

Kirkpatrick, J.D., Reid, I.N., Liebert, J., Cutri, R.M., Nelson, B., Beichman, C.A., Dahn, C.C., Monet, D.G., Gizis, J.E. \& Skrutskie, M.F. 1999, ApJ, 519, 834

Leggett, S.K. 1992, ApJS, 82, 351

Leggett, S.K., Allard, F. Berriman, G., Dahn, C.C. \& Hauschildt, P.H. 1996, ApJS, 104, 117

Leggett, S.K., Toomey, D.W., Geballe, T.R. \& Brown, R.H. 1999, ApJ, 517, L139

Liebert, J., Reid, I.N., Burrows, A., Burgasser, A.J., Kirkpatrick, J.D. \& Gizis, E. 2000, ApJ, in press (astro-ph/0003068)

Lodders, K. 1999, ApJ, 519793

Marley, M.S., Saumon, D., Guillot, T., Freedman, R.S., Hubbard, W.B., Burrows, A., \& Lunine, J.I. 1996, Science, 272, 1919

Matthews, K., Nakajima, T., Kulkarni, S.R. \& Oppenheimer, B.R. 1996, AJ, 112, 1678
Mountain, C.M., Robertson, D.J., Lee, T.J. \& Wade, R. 1990, Proc. SPIE, 1235, 25

Nakajima, T., Oppenheimer, B.R., Kulkarni, S.R., Golimowski, D.A., Matthews, K., \& Durrance, S.T. 1995, Nature, 378, 463

Noll, K.S., Geballe, T.R. \& Marley, M.S. 1997, ApJ, 489, L87

Oppenheimer, B.R., Kulkarni, S.R., Matthews, K. \& Nakajima, T. 1995, Science, 270, 1478

Oppenheimer, B.R., Kulkarni, S.R., Matthews, K. \& van Kerkwijk, M.H. 1998, ApJ, 502, 932

Partridge, H. \& Schwenke, D.W. (1997) J. Chem. Phys. 106, 4618

Perryman et al. 1997, A\&A, 323, L49

Rothman, L.S., et al. 1998, J. Quant. Spec. Radiat. Transf., 60, 665

Schiavon, R.P., Barbuy, B., \& Singh, P.D. 1997, ApJ, 484, 499

Schultz, A.B., et al. 1998, ApJ, 492, L181

Strauss, M.A. et al. 1999, ApJ, 552, L61

Strong, K., Taylor, F.W., Calcutt, S.B., Remedios, J.J. \& Ballard, J. 1993, JQSRT, 50363

Tsuji, T., Ohnaka, K., \& Aoki, W. 1996a, A\&A, 305, L1

Tsuji, T., Ohnaka, K., Aoki, W. \& Nakajima, T. 1996b, A\&A, 308, L29

Tsuji, T., Ohnaka, K., \& Aoki, W. 1999, ApJ, 520, L119

This 2-column preprint was prepared with the AAS LATEX macros v5.0. 
Fig. 1.- $J$ band spectrum. The spectral resolution is $R=2400$ and the noise level at the maximum flux level is indicated by the error bar. Nearly all features in this spectrum are caused by $\mathrm{H}_{2} \mathrm{O}$ absorption, except at short wavelengths where the $\mathrm{CH}_{4}$ opacity becomes significant, and for two strong $\mathrm{K}$ I lines. The flux calibration is approximate.

Fig. 2.- Same as Fig. 1 for the $H$ band. The features seen in the spectrum are caused by $\mathrm{H}_{2} \mathrm{O}$ for $\lambda \lesssim 1.59 \mu \mathrm{m}$. At longer wavelengths, the rapid decrease in flux corresponds to the blue side of a strong $\mathrm{CH}_{4}$ absorption band. Both $\mathrm{NH}_{3}$ and $\mathrm{H}_{2} \mathrm{~S}$ have significant opacity in this spectral range but show no distinctive feature at this resolution. The flux calibration is approximate.

Fig. 3.- Same as Fig. 1 for the $K$ band. The features seen in the spectrum are caused by $\mathrm{H}_{2} \mathrm{O}$ for $\lambda<2.11 \mu \mathrm{m}$. At longer wavelengths, the rapid decrease in flux corresponds to the blue side of a strong $\mathrm{CH}_{4}$ absorption band. Several strong $\mathrm{NH}_{3}$ features are seen shortward of $2.06 \mu \mathrm{m}$. Models predict a single $\mathrm{H}_{2} \mathrm{~S}$ absorption feature at $2.1084 \mu \mathrm{m}$. The flux calibration is approximate.

Fig. 4.- Evolution of solar metallicity brown dwarfs and giant planets in the effective temperature - gravity plane. The heavy solid lines are cooling tracks for objects with masses of 0.075 , $0.07,0.06,0.05,0.04,0.03,0.02$, and $0.01 M_{\odot}$, from top to bottom, respectively (Burrows et al. 1997). Evolution proceeds from right to left and isochrones (dotted lines) are labeled with the age in Gyr. The band crossing the center of the figure is the locus of all models with the luminosity of Gl $229 \mathrm{~B}\left(L_{\text {bol }}=6.2 \pm 0.55 \times 10^{-6} L_{\odot}\right)$. Filled symbols correspond to the models listed in Table 2 and used for the present analysis.

Fig. 5.- Temperature-pressure profiles of the three atmosphere models used in this analysis. Labels correspond to the models shown in Fig. 5 and Table 2 .

Fig. 6.- The temperature and pressure at the photosphere $\left(\tau_{\lambda}=2 / 3\right)$ as a function of wavelength for model B $\left(T_{\text {eff }}=940 \mathrm{~K}, \log g=5\right)$. The temperature plotted (left axis) is equivalent to a brightness temperature. Horizontal bars indicate the wavelength range of $\mathrm{NH}_{3}$ (solid lines) and $\mathrm{CO}$ (dotted lines) bands. The short vertical bar indicates a $\mathrm{H}_{2} \mathrm{~S}$ absorption feature at $2.1084 \mu \mathrm{m}$ (see $\S 5.4 .2)$.

Fig. 7.- A spectrum computed with model B, $[\mathrm{M} / \mathrm{H}]=-0.3$, and dust opacity (thin line) is compared to the observed spectrum of Oppenheimer et al. (1998) (heavy line). Top panel: Entire fitted region. Bottom panel: details of the $\mathrm{H}_{2} \mathrm{O}$ absorption band at a resolution $R=2250$.

Fig. 8.- Top panel: Effect of the metallicity on the $J$ band spectrum. Data are compared to two model B spectra with $[\mathrm{M} / \mathrm{H}]=-0.4$ and 0 . Bottom panel: The best fit for model $\mathrm{B}$ is obtained for $[\mathrm{M} / \mathrm{H}]=-0.2$. See text. The synthetic spectra are normalized to the observations at $1.2472 \mu \mathrm{m}$ and are plotted at the same resolution $(R=2400)$.

Fig. 9.- Effect of the $\mathrm{NH}_{3}$ abundance on the $H$ band spectrum. The spectrum is computed for model $B$ with $[\mathrm{M} / \mathrm{H}]=-0.3$ with the chemical equilibrium abundance of $\mathrm{NH}_{3}$ (dotted line) and without $\mathrm{NH}_{3}$ opacity (solid line). The dotted curve represents our best fit for this value of $[\mathrm{M} / \mathrm{H}]$. The synthetic spectra are normalized to the observations at $1.5744 \mu \mathrm{m}$ and plotted at the same resolution $(R=2100)$.

Fig. 10.- Top panel: Effect of the metallicity on the $H$ band spectrum. Data are compared to two model B spectra with $[\mathrm{M} / \mathrm{H}]=-0.4$ and 0 . Bottom panel: The best fit for model $\mathrm{B}$ is obtained for $[\mathrm{M} / \mathrm{H}]=-0.3$. In all cases, the $\mathrm{NH}_{3}$ abundance is determined from the chemical equilibrium. See text. See Fig. 9 caption for additional details.

Fig. 11. - Effect of the $\mathrm{H}_{2} \mathrm{~S}$ abundance on the $H$ band spectrum. The spectra are computed for model $\mathrm{B}$ with $[\mathrm{M} / \mathrm{H}]=-0.3$ with the chemical equilibrium abundance of $\mathrm{H}_{2} \mathrm{~S}$ and without $\mathrm{H}_{2} \mathrm{~S}$ opacity. See Fig. 9 caption for additional details.

Fig. 12. - Carbon monoxide absorption band in the $H$ band. The spectra are computed for model $\mathrm{B}$ with $[\mathrm{M} / \mathrm{H}]=0$ with the chemical equilibrium abundance of $\mathrm{CO}$ (solid line) and with the CO abundance equal to the elemental carbon abundance (dotted line). A spectrum computed with the $\mathrm{CO}$ abundance set to zero overlaps the equilibrium CO curve. See Fig. 9 caption for additional details. 
Fig. 13. - Synthetic spectrum for model B with $[\mathrm{M} / \mathrm{H}]=-0.3$ compared to the observed $K$ band spectrum. The synthetic spectrum is normalized to the data at $2.0513 \mu \mathrm{m}$ and plotted at the same resolution $(R=2800)$.

Fig. 14.- Top panel: Effect of the metallicity on the $K$ band spectrum. Data are compared to two model B spectra with $[\mathrm{M} / \mathrm{H}]=-0.5$ and 0 . Bottom panel: The best fit for model $\mathrm{B}$ is obtained for $[\mathrm{M} / \mathrm{H}]=-0.3$. All features in this figure are caused by $\mathrm{H}_{2} \mathrm{O}$. See Fig. 13 caption for additional details.

Fig. 15.- Portion of the $K$ band spectrum which shows $\mathrm{NH}_{3}$ features. The data are shown by the heavy solid line, with a representative error bar. Ammonia features are indicated by tick marks. Top panel: Synthetic spectrum computed from model B with $[\mathrm{M} / \mathrm{H}]=-0.3$ (dotted line) and after removing all $\mathrm{NH}_{3}$ opacity (thin solid line). Bottom panel: Same as above but the equilibrium $\mathrm{NH}_{3}$ abundance has been uniformly reduced by a factor of 0.25 throughout the atmosphere. See Fig. 13 caption for additional details.

Fig. 16.- Opacity of $\mathrm{H}_{2} \mathrm{~S}$ in the $2.11 \mu \mathrm{m}$ region for $T=800 \mathrm{~K}$ and $P=1$ bar. The spectral resolution is $R=4.8 \times 10^{5}$. Synthetic spectra predict that the strong feature at $2.1084 \mu \mathrm{m}$ should be observable.

Fig. 17. - Hydrogen sulfide feature predicted at $2.1084 \mu \mathrm{m}$. The observed spectrum is shown by a heavy solid line. The thin lines in each panel show spectra computed for the indicated model (A, B, and C). Each spectrum is computed with the best-fitting metallicity for that model (Table 2 ). In each panel, the upper curve is calculated without $\mathrm{H}_{2} \mathrm{~S}$ opacity, and the lower curve is computed with the chemical equilibrium abundance of $\mathrm{H}_{2} \mathrm{~S}$ for the chosen metallicity. For model B (middle panel), the effect of reducing the equilibrium $\mathrm{H}_{2} \mathrm{~S}$ abundance by factors of 0.5 and 0.25 , respectively, is also shown. All synthetic spectra are normalized to the data at $2.1062 \mu \mathrm{m}$ and the resolution is $R=2800$.

Fig. 18. - Equilibrium chemistry in the atmosphere of Gl 229B. The curves show the abundance of the important molecular species as a function of depth for each of the three gravities, computed with the metallicity we have determined for each
(Table 2). Depth increases toward the right along the ordinate axis and is indicated by both temperature and pressure scales. The extent of the convection zone is shown by the shaded region. Horizontal bars at the top show the regions probed by spectroscopic observations of various molecular bands. The top row of solid bars shows three regions where $\mathrm{NH}_{3}$ is detectable: $10 \mu \mathrm{m}$ region and the $K$ and $H$ bands, from left to right, respectively. Dotted bars show the CO bands at $4.7 \mu \mathrm{m}$ and in the $H$ band, respectively. The vertical tick shows the depth where the $2.1084 \mu \mathrm{m}$ feature of $\mathrm{H}_{2} \mathrm{~S}$ is formed. The dotted box shows the $\mathrm{CO}$ abundance determined from the at $4.7 \mu \mathrm{m}$ band spectra of Noll et al. (1997) and Oppenheimer et al. (1998). Our determinations of the $\mathrm{NH}_{3}$ abundance in the $K$ an $H$ bands are shown by vertical boxes, the height of the box indicating the estimated uncertainty. For the $K$ band measurements, an arrow indicates that the $\mathrm{NH}_{3}$ abundance could be close to zero. The labeling of the panels corresponds to the entries in Table 2.

Fig. 19.- Ammonia features in the $10 \mu \mathrm{m}$ region. The spectra are computed with model B and $[\mathrm{M} / \mathrm{H}]=-0.4$. Each curve corresponds to a different depletion of $\mathrm{NH}_{3}$ relative to its equilibrium abundance, the abundance of all other compounds being kept the same. The equilibrium abundance of $\mathrm{NH}_{3}$ has been multiplied by $0,0.25,0.5$, and 1 , from top to bottom, respectively. All spectra are normalized at $10 \mu \mathrm{m}$ and the resolution is $R=200$. 
TABLE 1

OBSERVING LOG

\begin{tabular}{cccccc}
\hline \hline UT Date & $\begin{array}{c}\text { wavelength } \\
(\mu \mathrm{m})\end{array}$ & $\begin{array}{c}\text { Resolving } \\
\text { Power }\end{array}$ & $\begin{array}{c}\text { Integ. Time } \\
\text { (minutes) }\end{array}$ & $\begin{array}{c}\text { mean airmass } \\
\text { Gl 229B }\end{array}$ & $\begin{array}{c}\text { mean airmass } \\
\text { BS 1849 }\end{array}$ \\
\hline 19980125 & $2.10-2.18$ & 3200 & 80 & 1.37 & 1.39 \\
19980125 & $2.02-2.10$ & 3100 & 93 & 1.40 & 1.35 \\
19980126 & $1.53-1.61$ & 2350 & 53 & 1.46 & 1.39 \\
19980126 & $1.25-1.30$ & 3000 & 53 & 1.33 & 1.32 \\
19980126 & $1.20-1.25$ & 2900 & 53 & 1.41 & 1.40 \\
19980127 & $2.02-2.10$ & 3100 & 93 & 1.36 & 1.32 \\
\hline
\end{tabular}

TABLE 2

OPTIMAL PARAMETERS

\begin{tabular}{|c|c|c|c|c|c|c|c|c|c|}
\hline \multirow[t]{2}{*}{ Model } & \multirow{2}{*}{$\begin{array}{l}\log g \\
(\mathrm{cgs})\end{array}$} & \multirow{2}{*}{$\begin{array}{l}T_{\text {eff }} \\
(\mathrm{K})\end{array}$} & \multicolumn{4}{|c|}{$[\mathrm{M} / \mathrm{H}]^{\mathrm{a}}$} & \multicolumn{3}{|c|}{$\mathrm{NH}_{3} /\left\{\mathrm{NH}_{3}\right\}_{\mathrm{eq}}$} \\
\hline & & & $0.92 \mu \mathrm{m}$ & $J$ & $H$ & $K$ & $H^{\mathrm{b}}$ & $H^{\mathrm{c}}$ & $K$ \\
\hline $\mathrm{A}$ & 4.5 & 870 & -0.5 & -0.5 & $\begin{array}{c}-0.3 \\
-0.4 \\
-0.5^{*}\end{array}$ & -0.5 & $\begin{array}{l}0 \\
0.25 \\
0.5\end{array}$ & $\begin{array}{l}0.25 \\
0.5 \\
1\end{array}$ & $\lesssim 0.40$ \\
\hline B & 5.0 & 940 & -0.3 & -0.2 & $\begin{array}{l}-0.1 \\
-0.2 \\
-0.3^{*}\end{array}$ & -0.3 & $\begin{array}{c}0 \\
0.25-0.5 \\
>0.5\end{array}$ & $\begin{array}{c}\lesssim 0.25 \\
0.5 \\
1\end{array}$ & $\lesssim 0.40$ \\
\hline $\mathrm{C}$ & 5.5 & 1030 & -0.2 & 0.1 & $\begin{array}{c}0.0 \\
-0.1^{*}\end{array}$ & -0.1 & $\begin{array}{l}0.5 \\
1\end{array}$ & $\begin{array}{l}0.5 \\
1\end{array}$ & $\lesssim 0.40$ \\
\hline
\end{tabular}

${ }^{\text {a }}$ An asterisk $(*)$ indicates the adopted metallicity.

${ }^{\mathrm{b}} \mathrm{H}_{2} \mathrm{~S}$ abundance set to 0 .

${ }^{\mathrm{c}} \mathrm{H}_{2} \mathrm{~S}$ abundance from chemical equilibrium.

TABLE 3

Abundance of CO FROM the $4.7 \mu \mathrm{m}$ Band

\begin{tabular}{ccrcc}
\hline \hline Model & $\begin{array}{c}\log g \\
(\operatorname{cgs})\end{array}$ & $\begin{array}{c}T_{\text {eff }} \\
(\mathrm{K})\end{array}$ & {$[\mathrm{M} / \mathrm{H}]$} & $\log X_{C O}$ \\
\hline $\mathrm{A}$ & 4.5 & 870 & -0.5 & $-4.0 \pm 0.25$ \\
$\mathrm{~B}$ & 5.0 & 940 & -0.3 & $-3.8 \pm 0.3$ \\
$\mathrm{C}$ & 5.5 & 1030 & -0.1 & $-3.5 \pm 0.3$ \\
\hline
\end{tabular}


TABLE 4

NeAR Infrared Colors of GL 229B

\begin{tabular}{|c|c|c|c|c|c|c|}
\hline Model & $\log g$ & $T_{\text {eff }}$ & {$[\mathrm{M} / \mathrm{H}]$} & $J-K$ & $H-K$ & $K-L^{\prime}$ \\
\hline $\mathrm{A}$ & 4.5 & 870 & -0.5 & -0.11 & -0.27 & 2.25 \\
\hline B & 5.0 & 940 & -0.3 & -0.14 & -0.30 & 2.26 \\
\hline $\mathrm{C}$ & 5.5 & 1030 & -0.1 & -0.08 & -0.24 & 2.17 \\
\hline \multicolumn{4}{|c|}{ Leggett et al. 1999} & $-0.10 \pm 0.07$ & $-0.07 \pm 0.07$ & $2.24 \pm 0.11$ \\
\hline
\end{tabular}




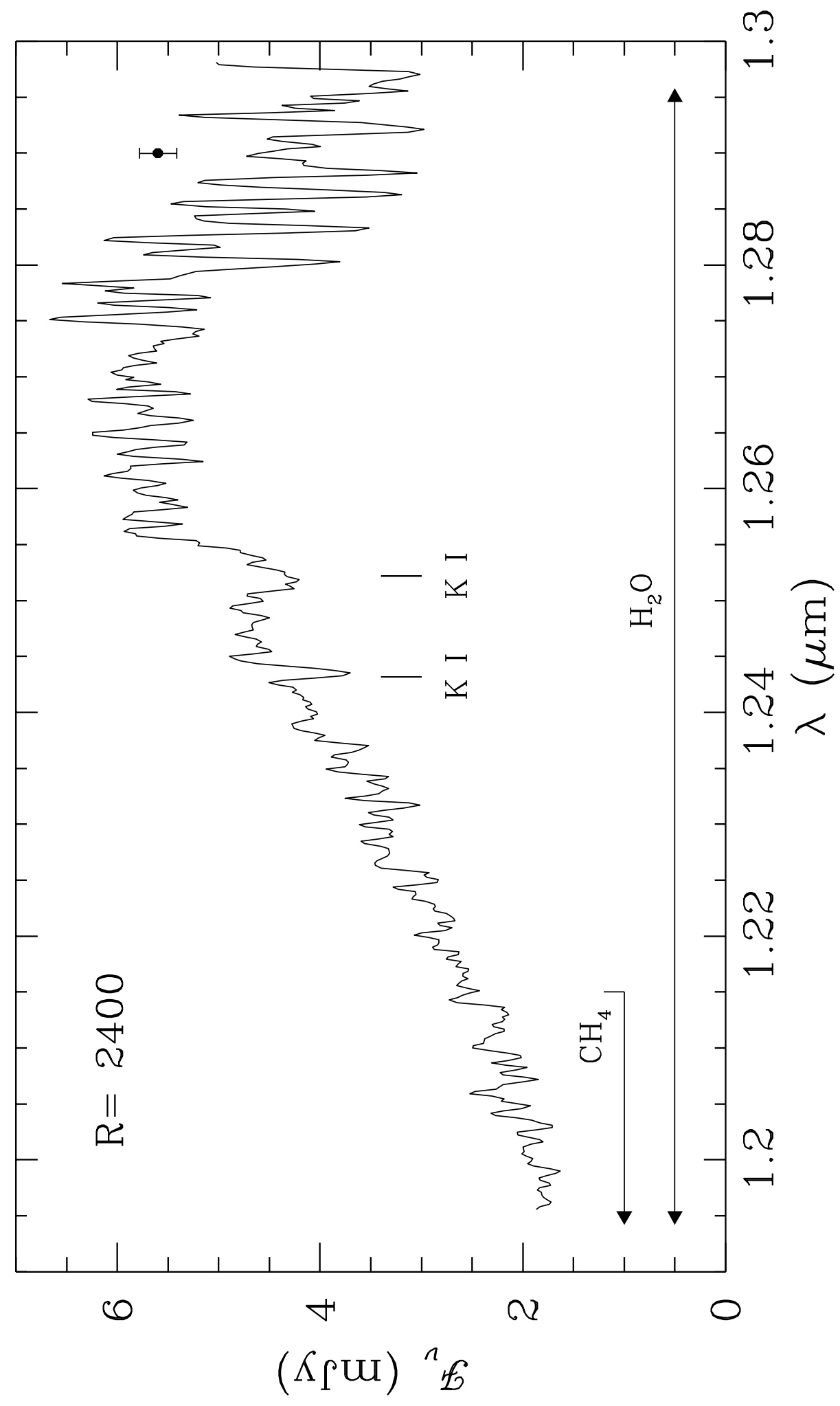




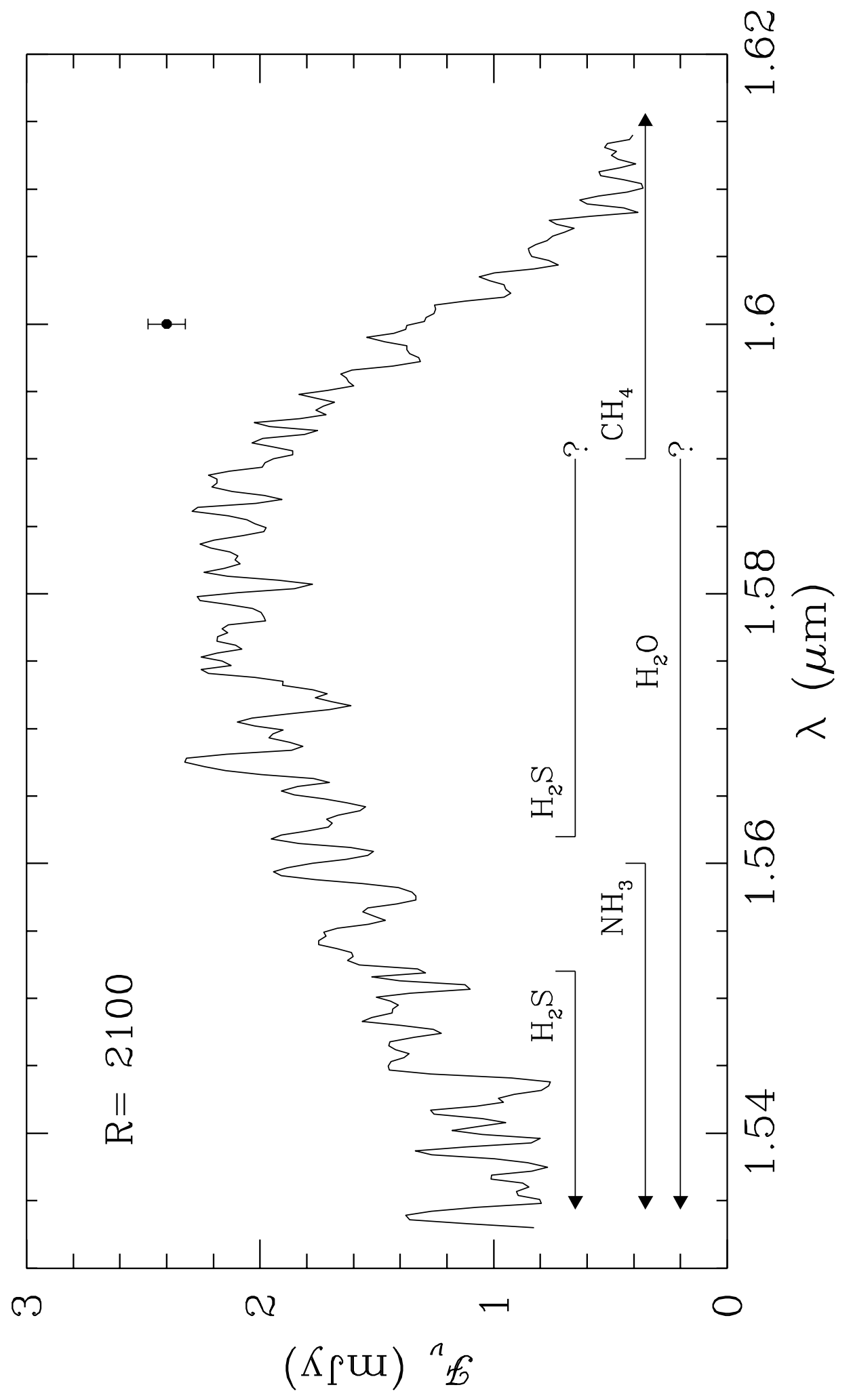




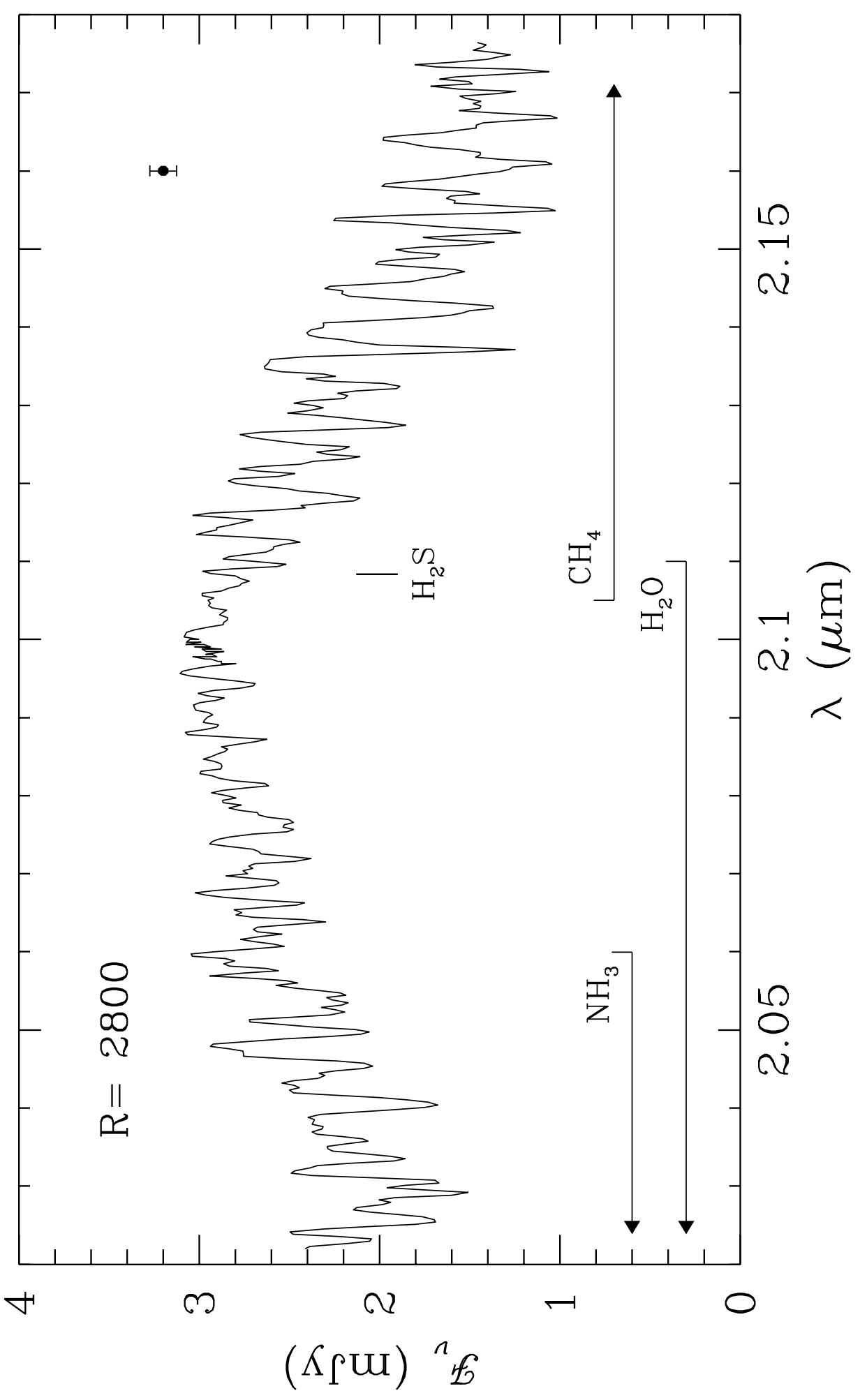




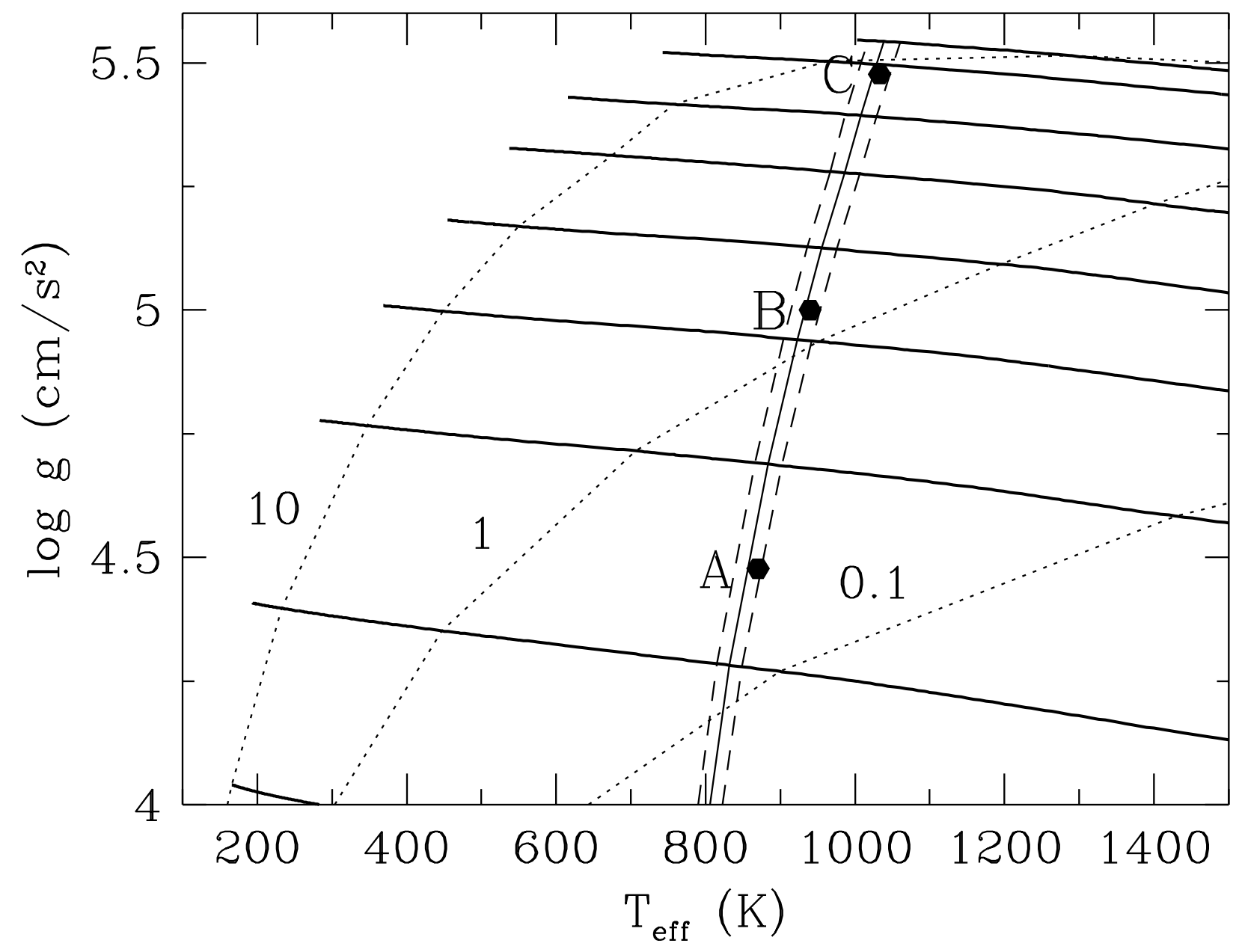




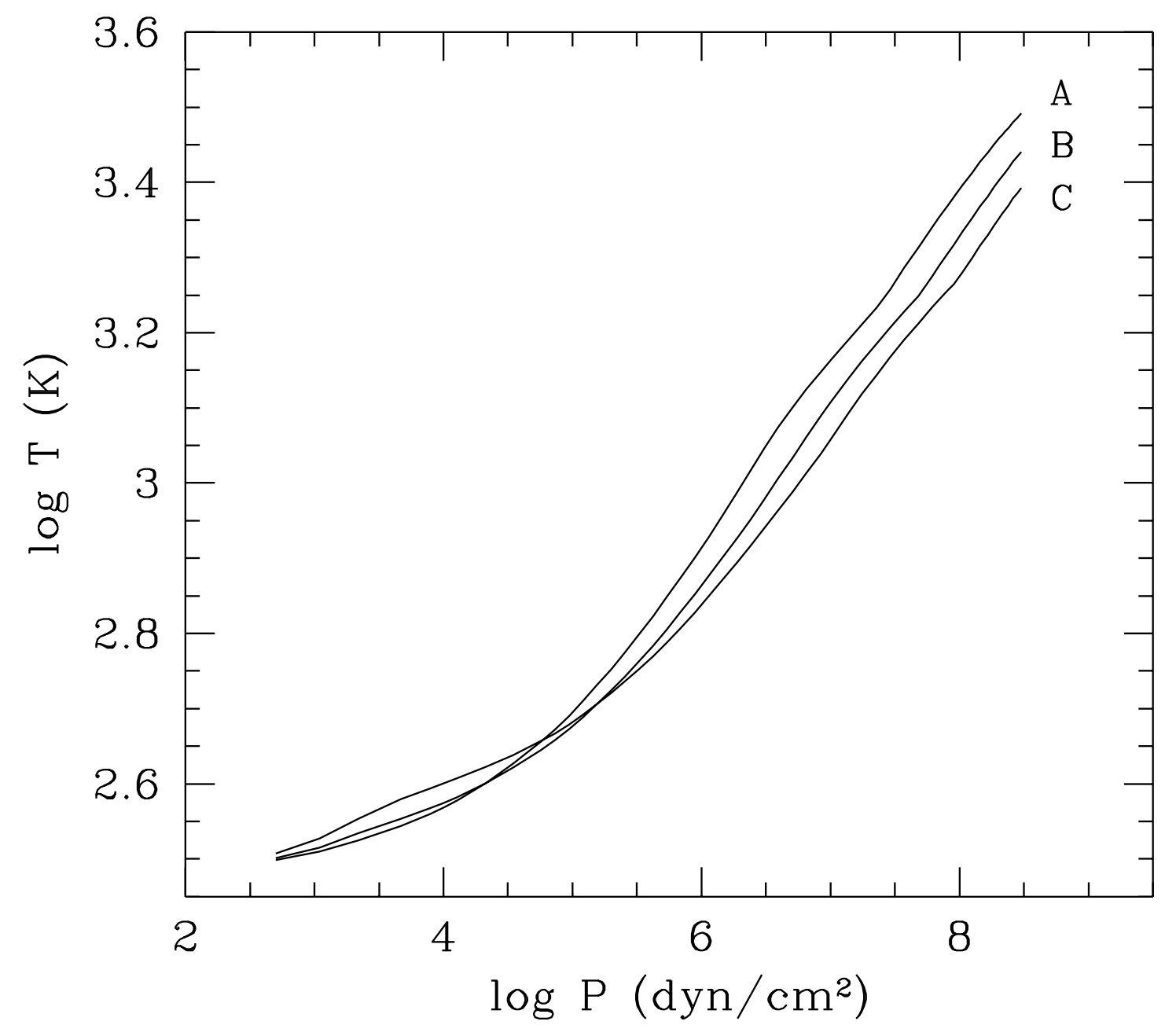




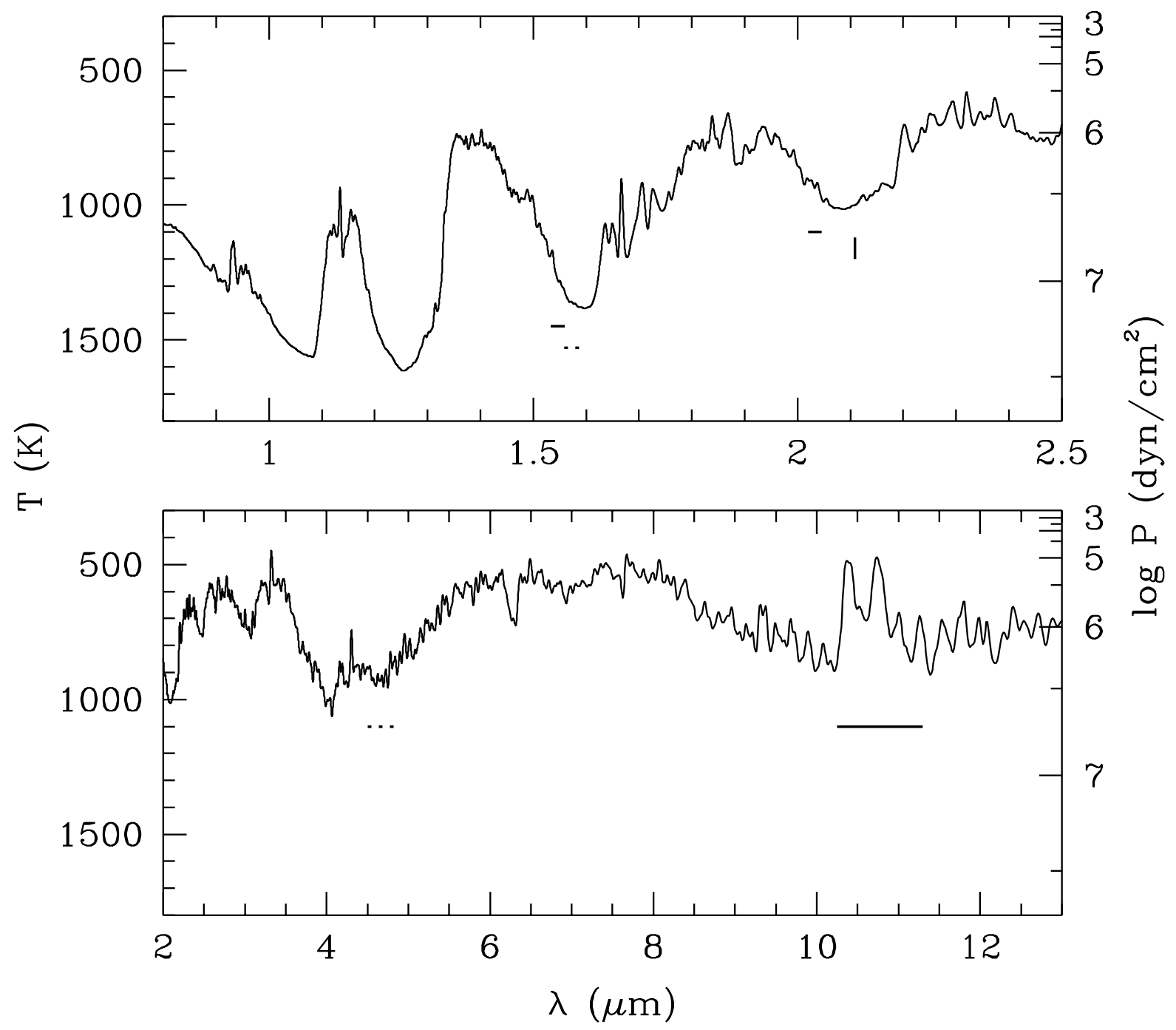



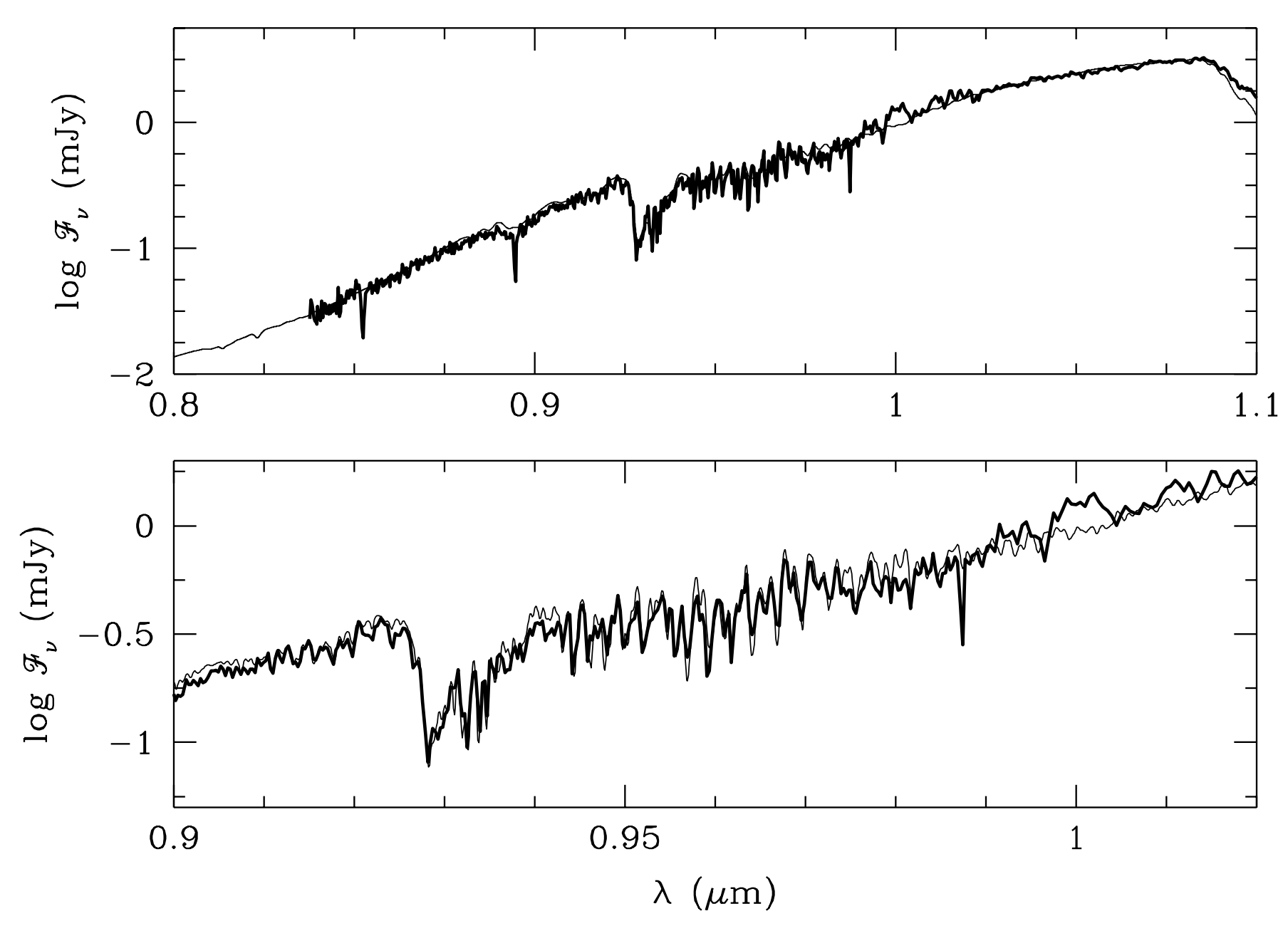




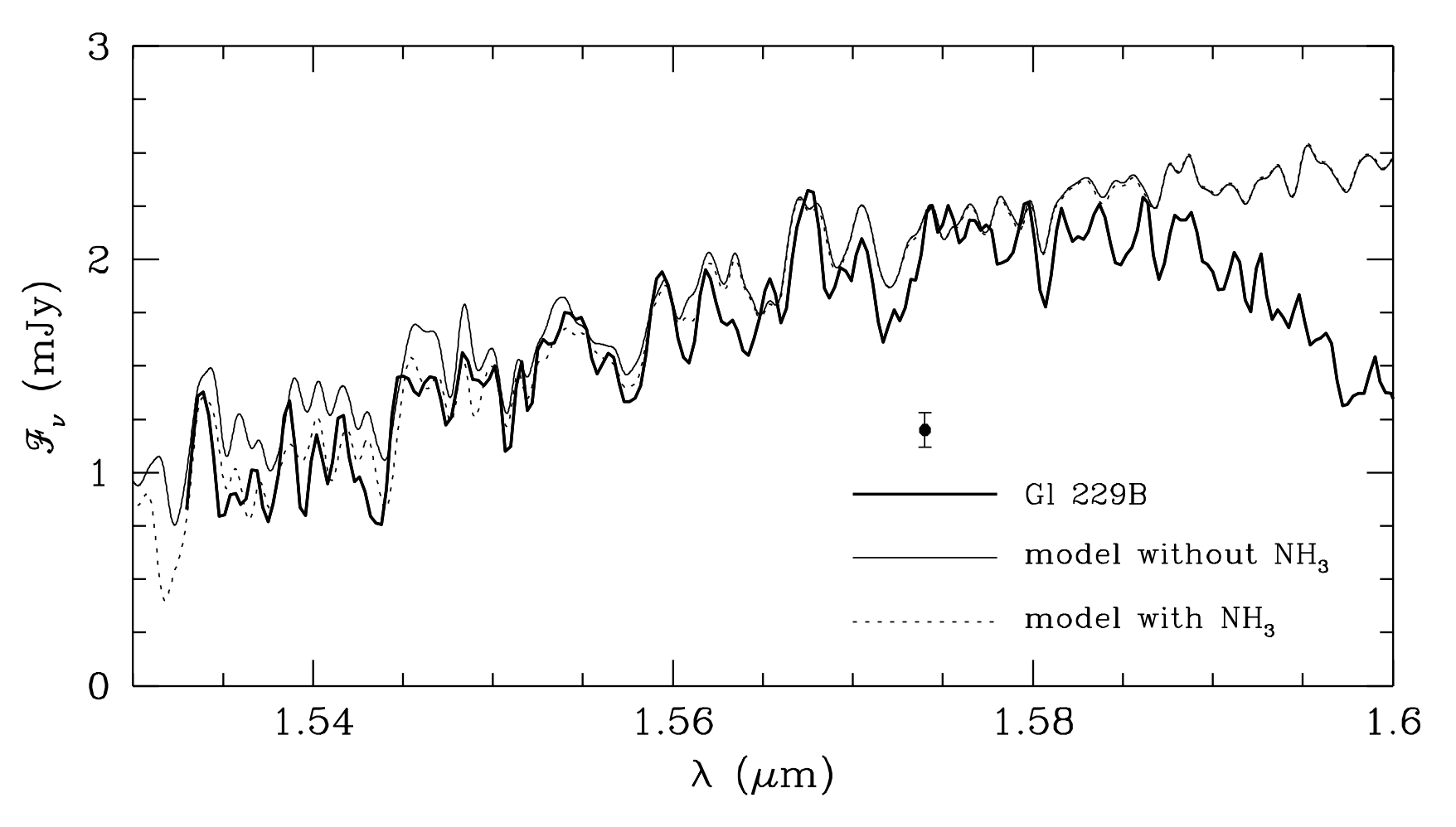




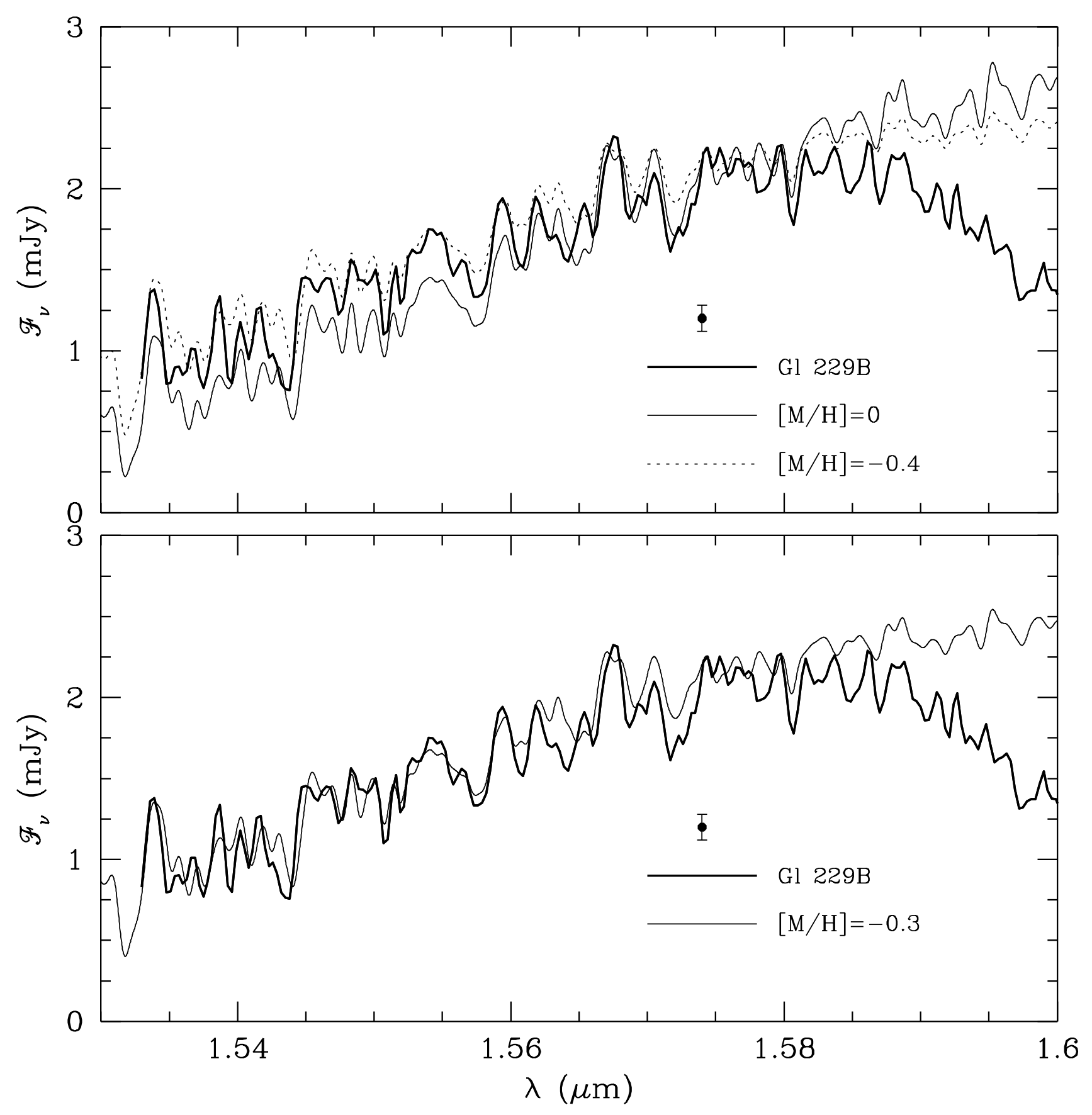




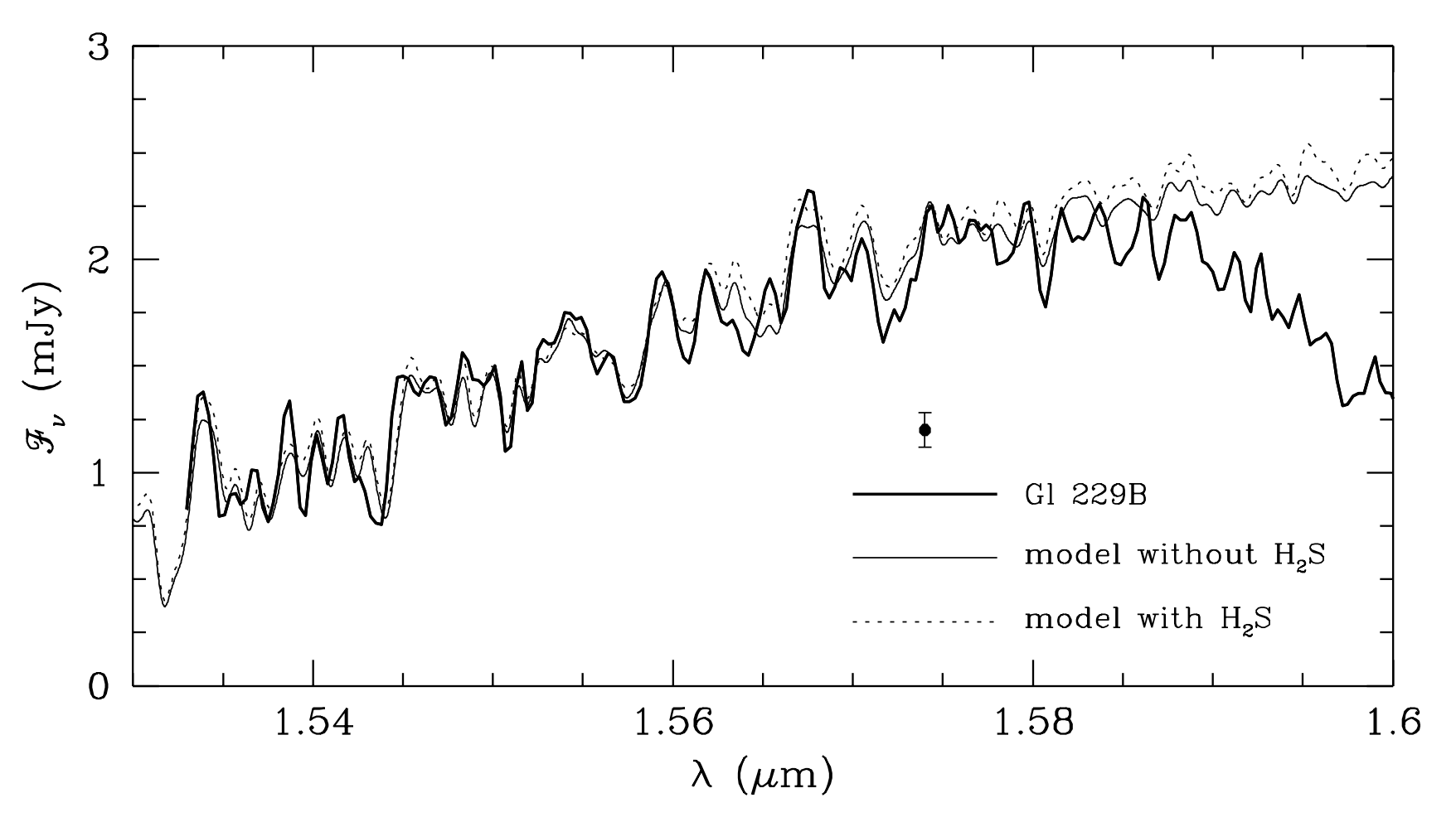




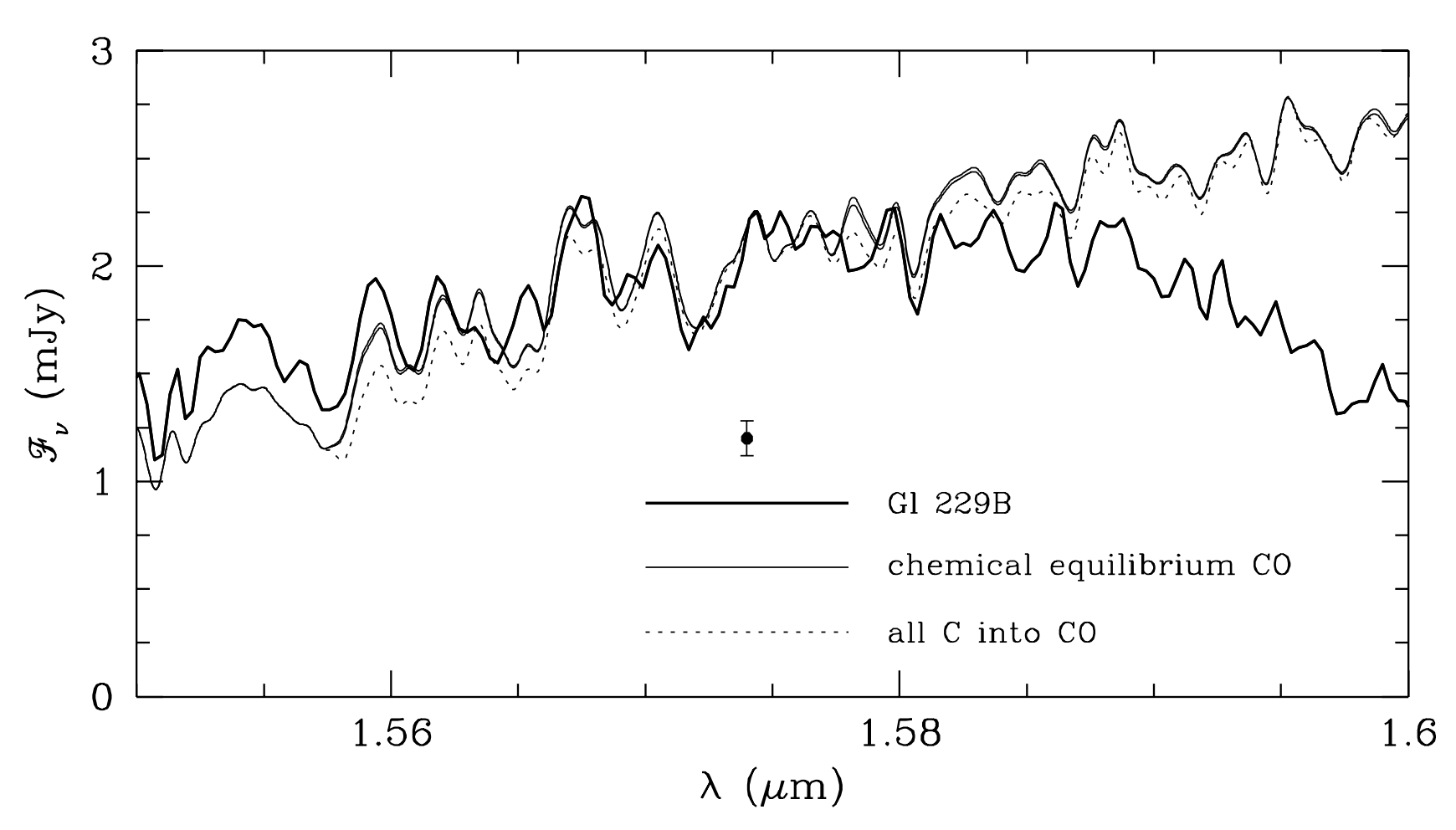




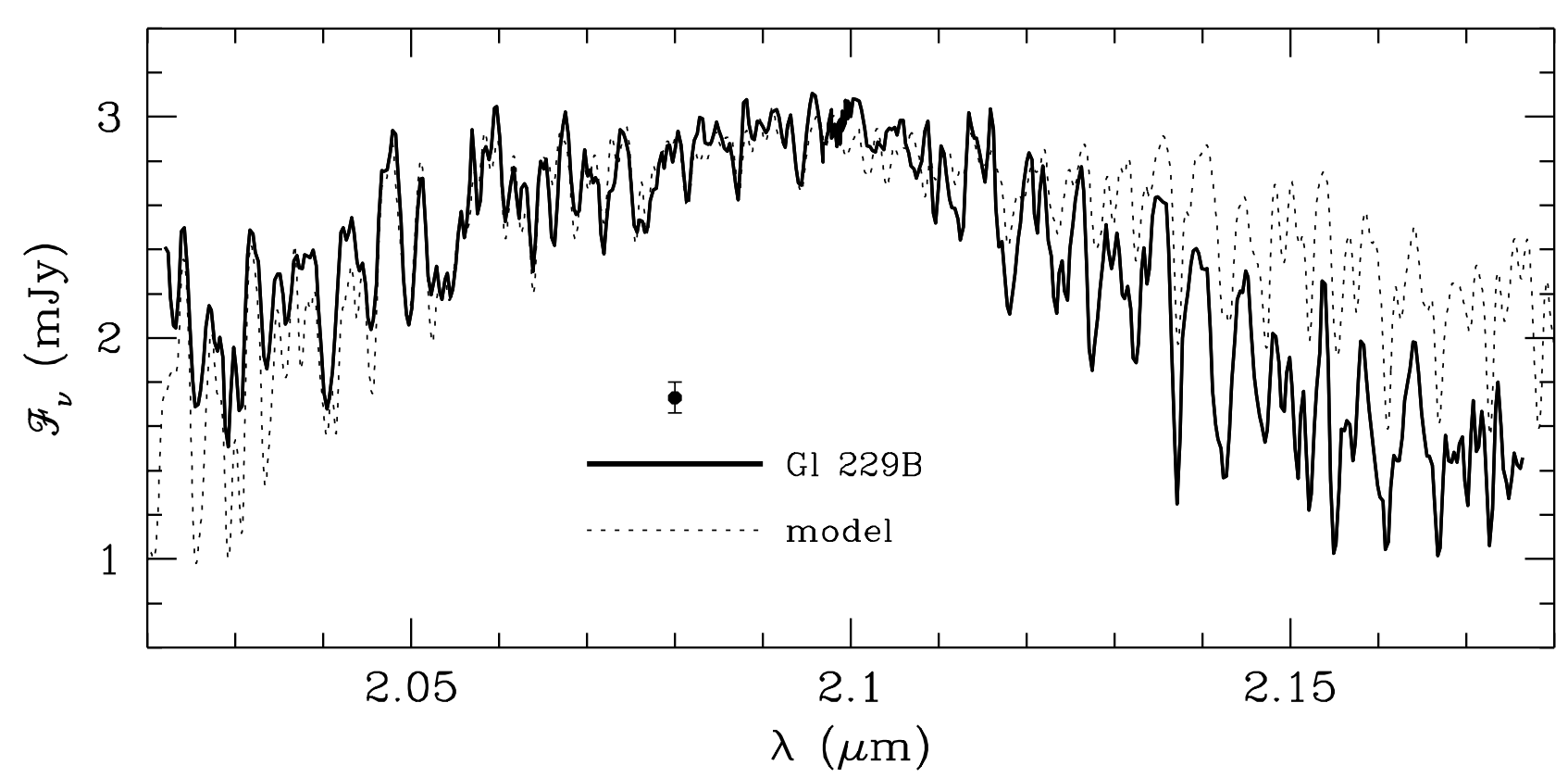



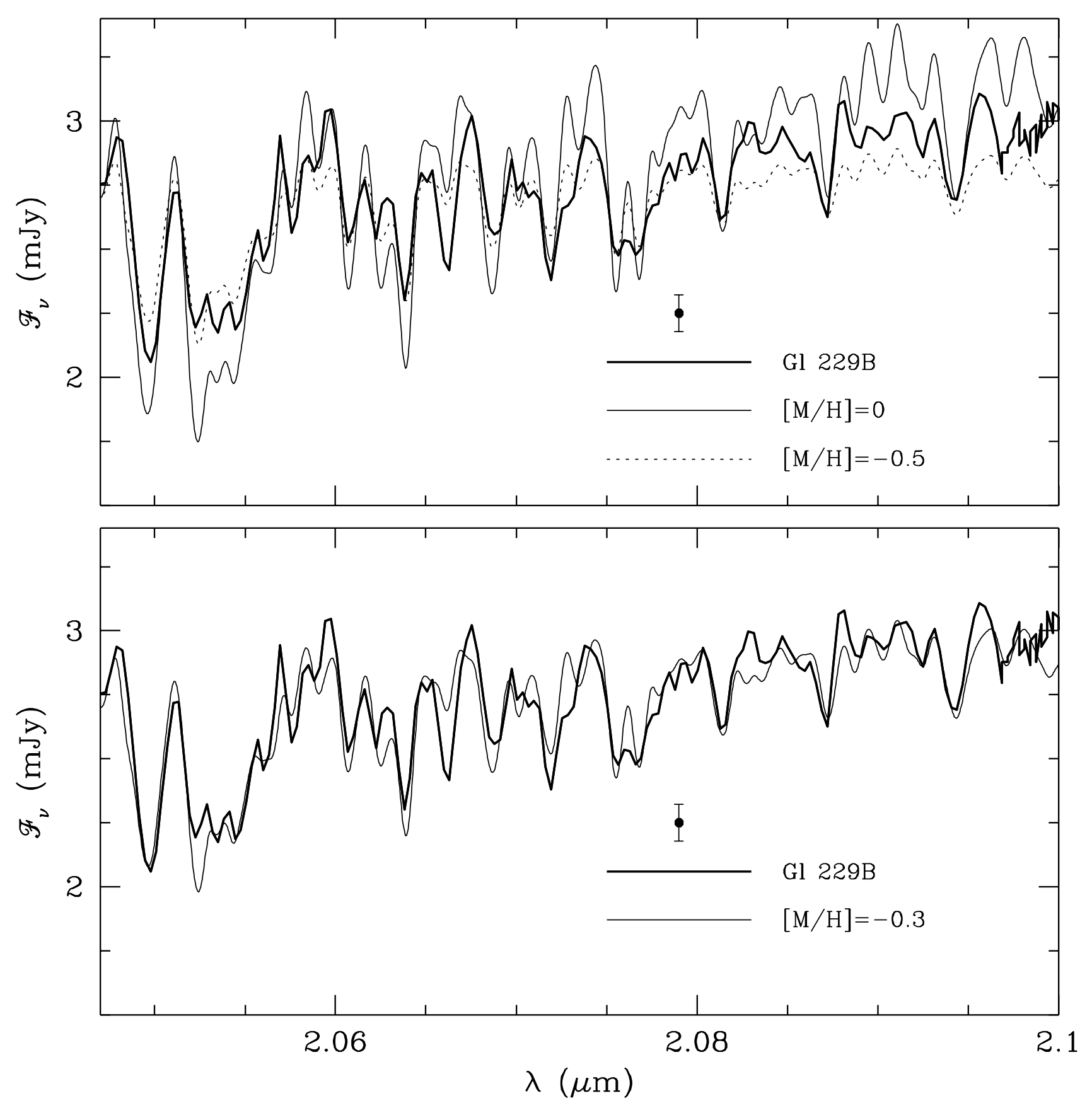

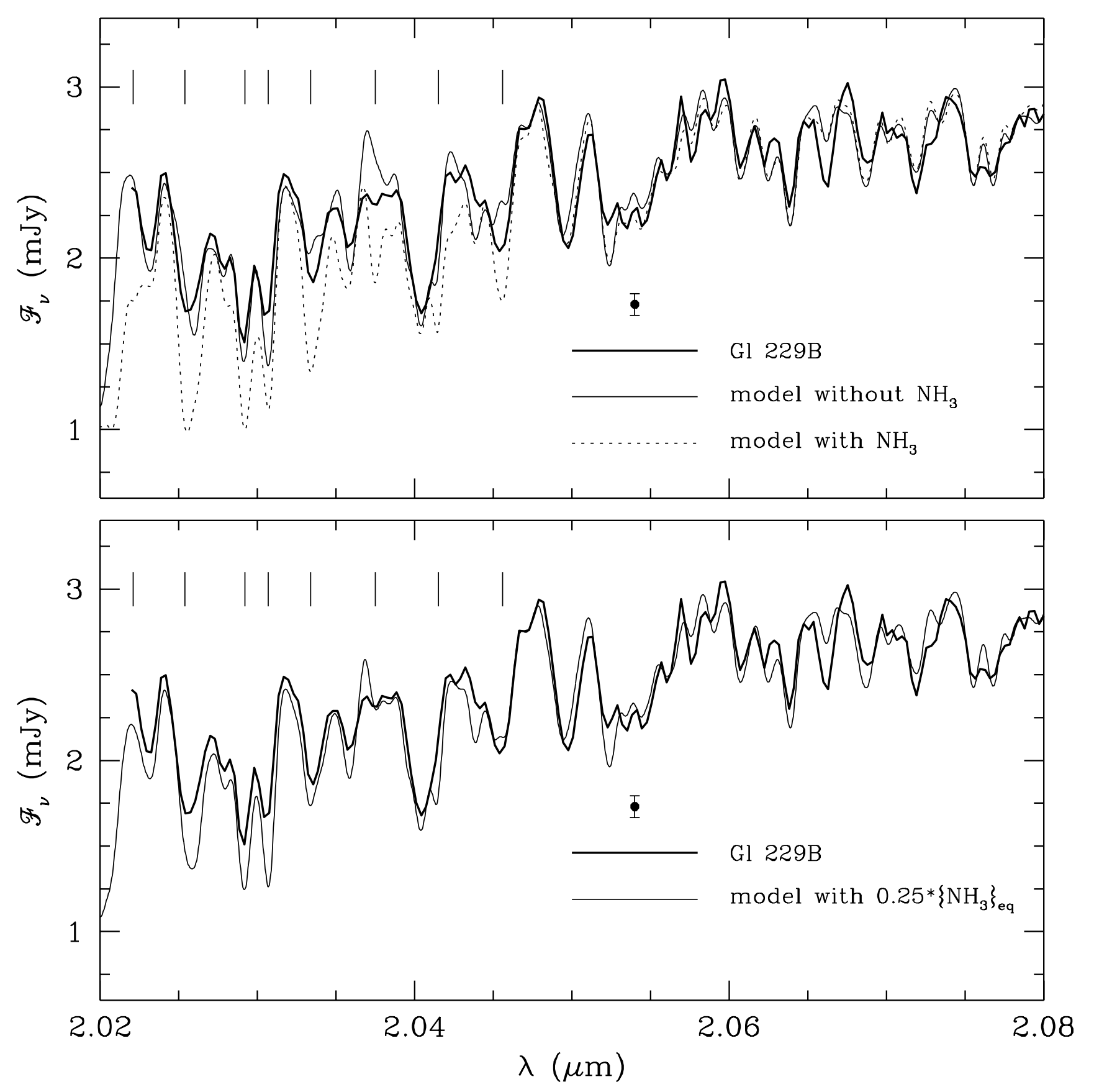


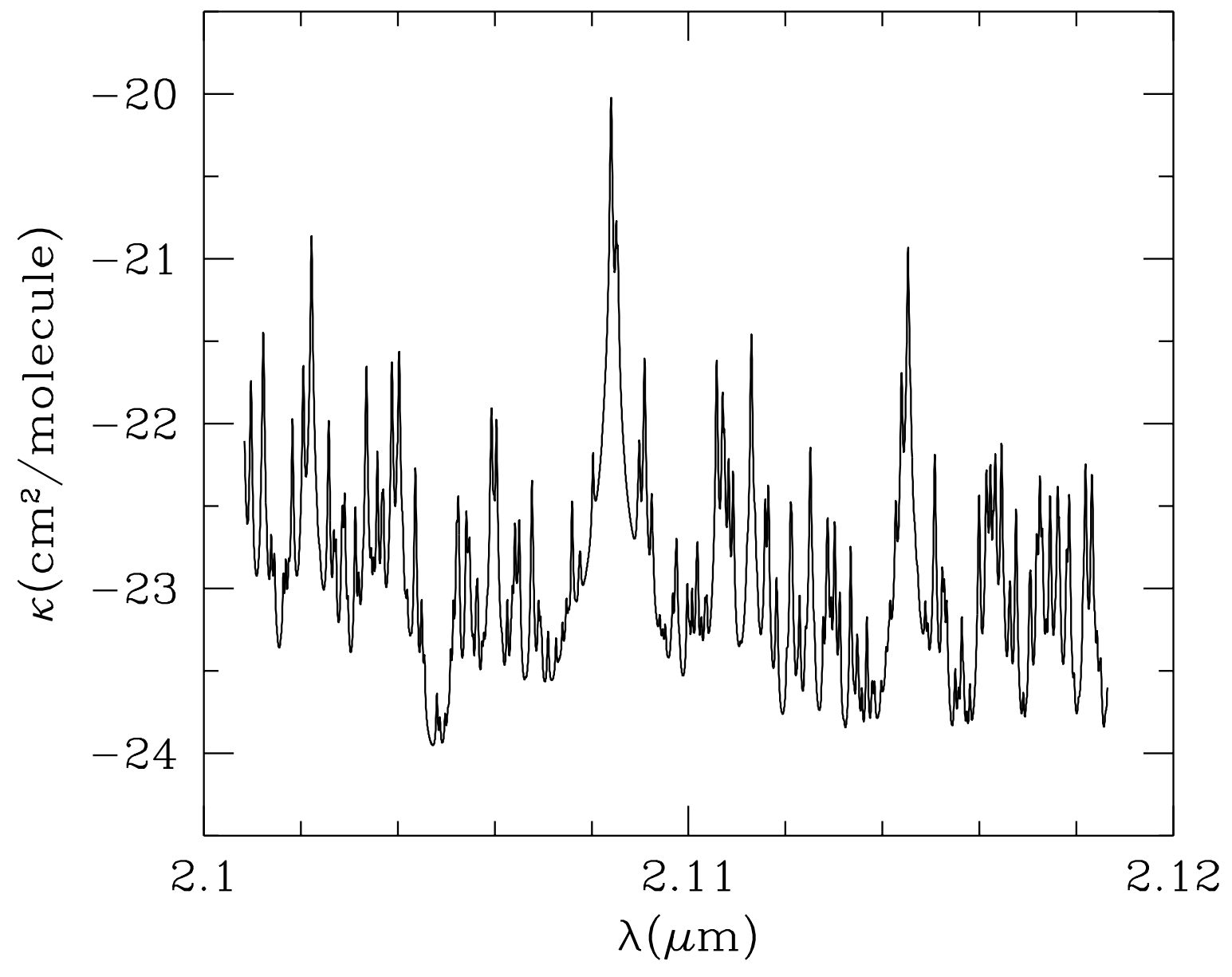




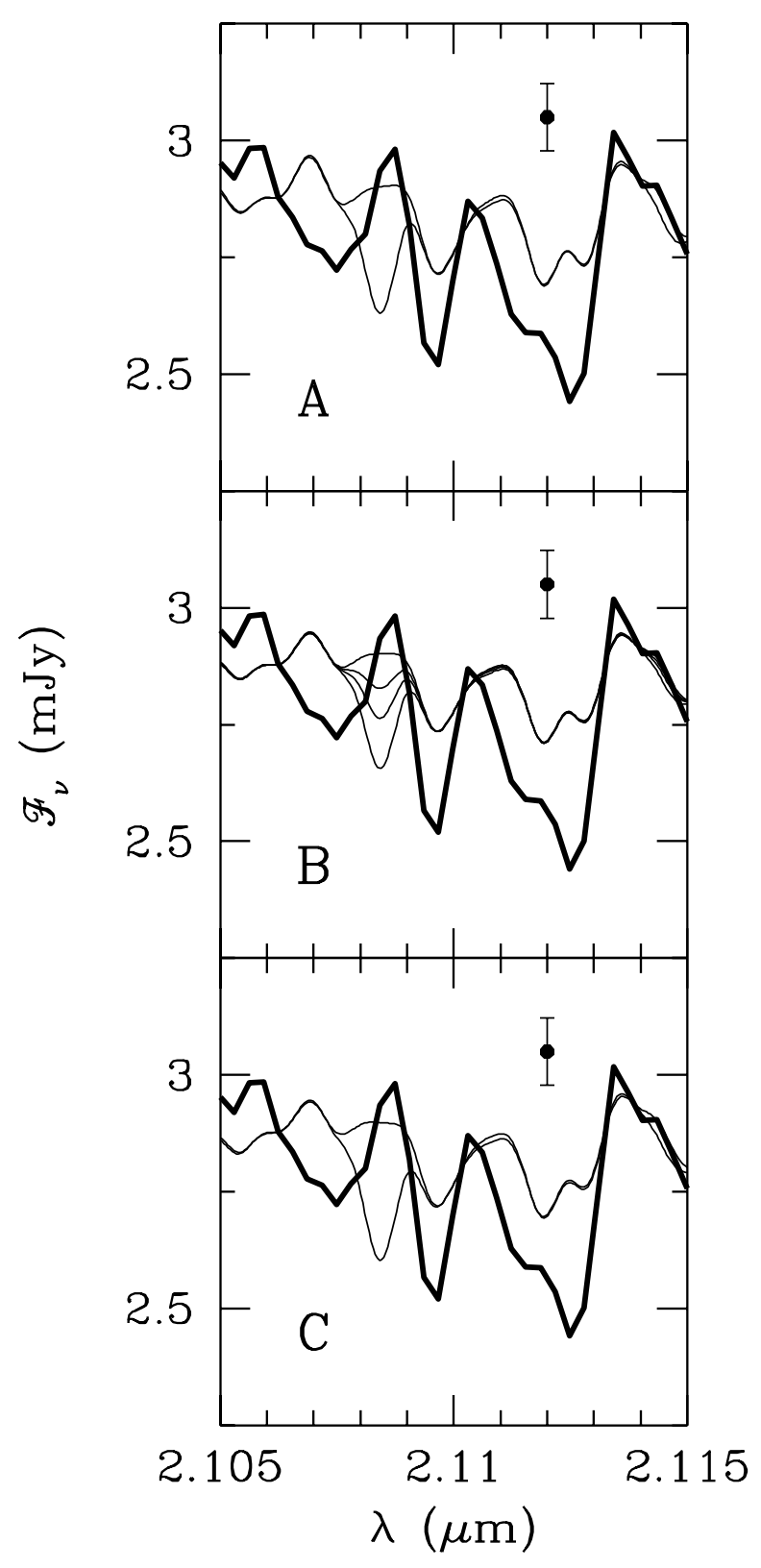




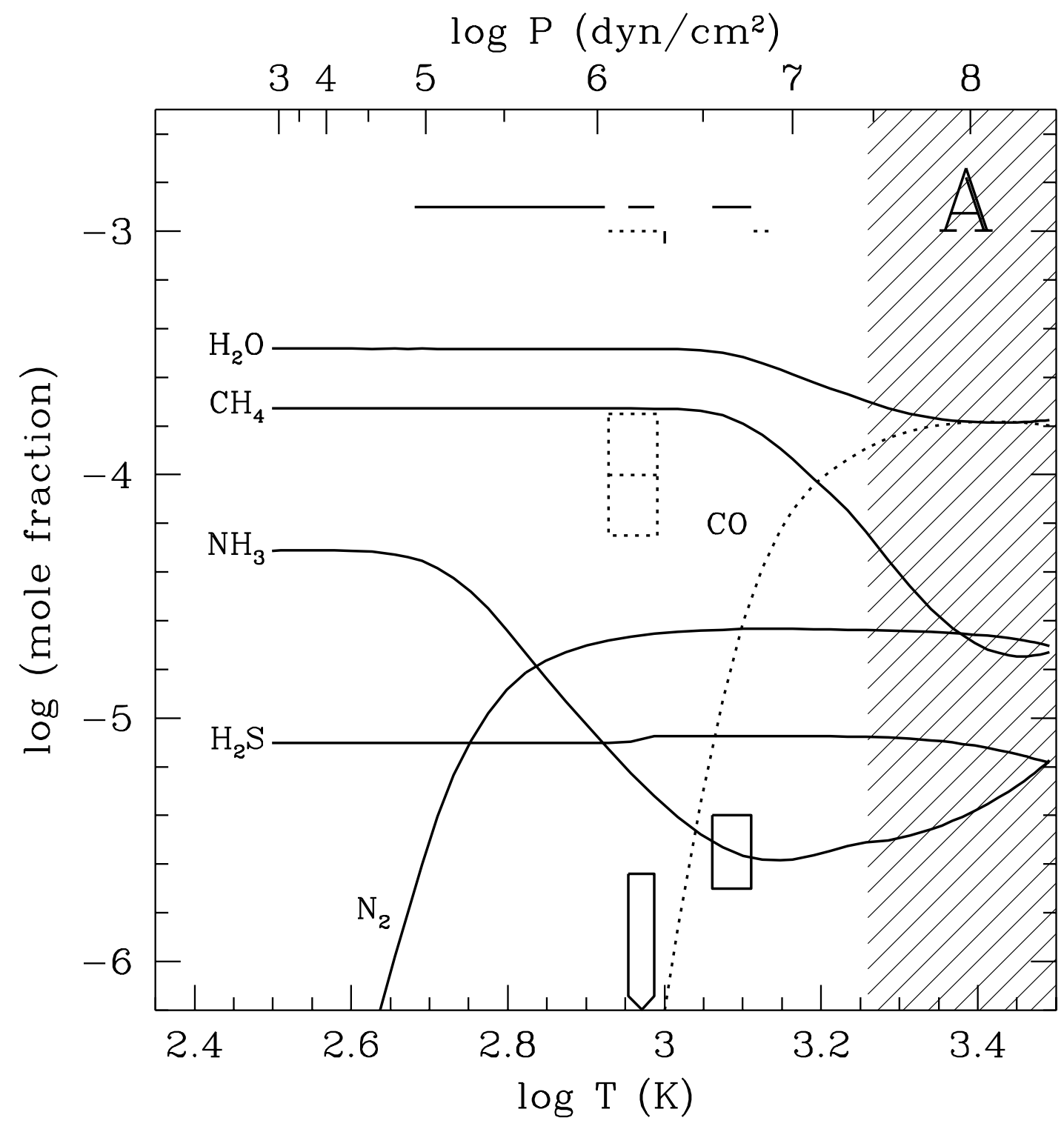




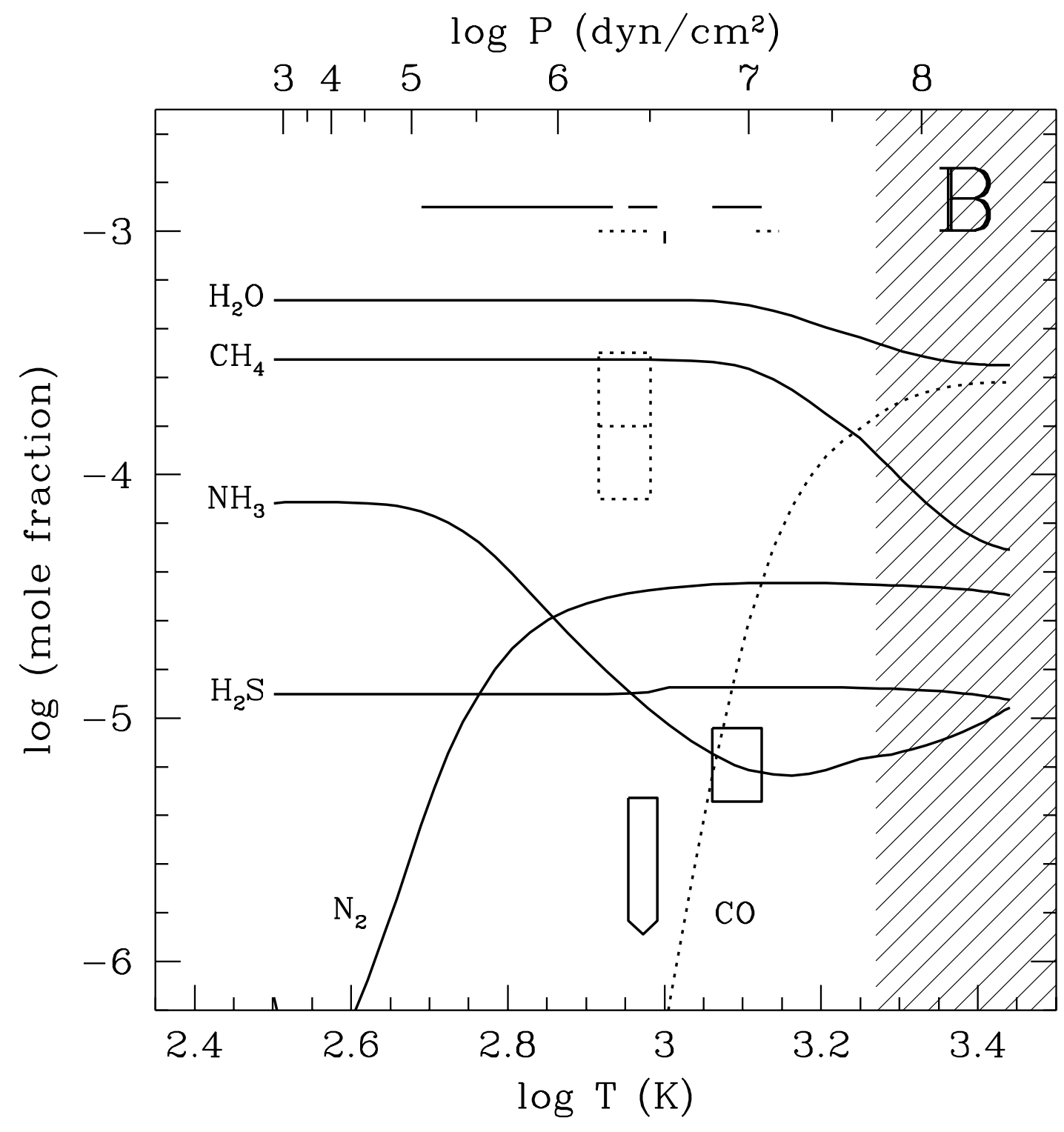




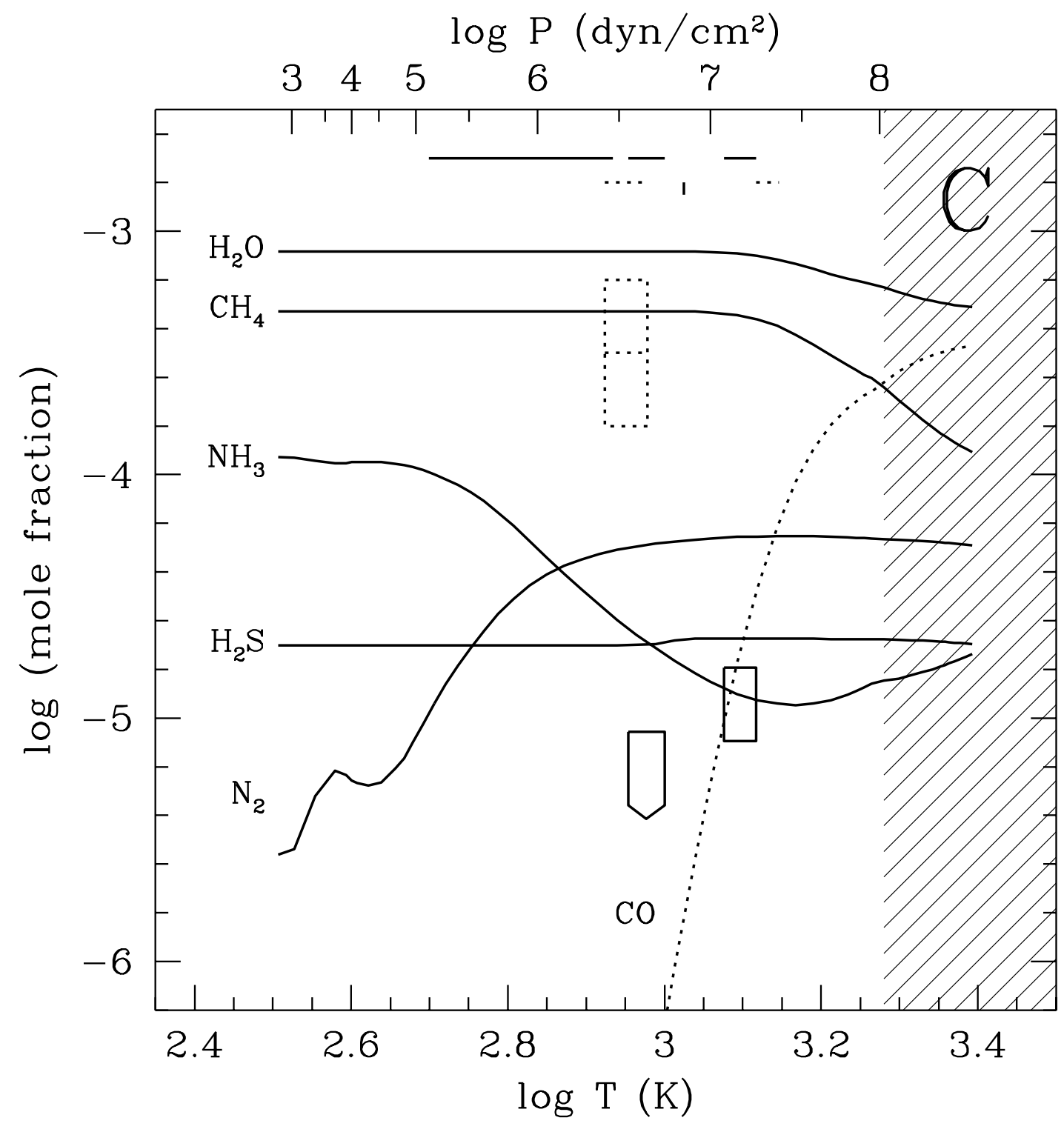




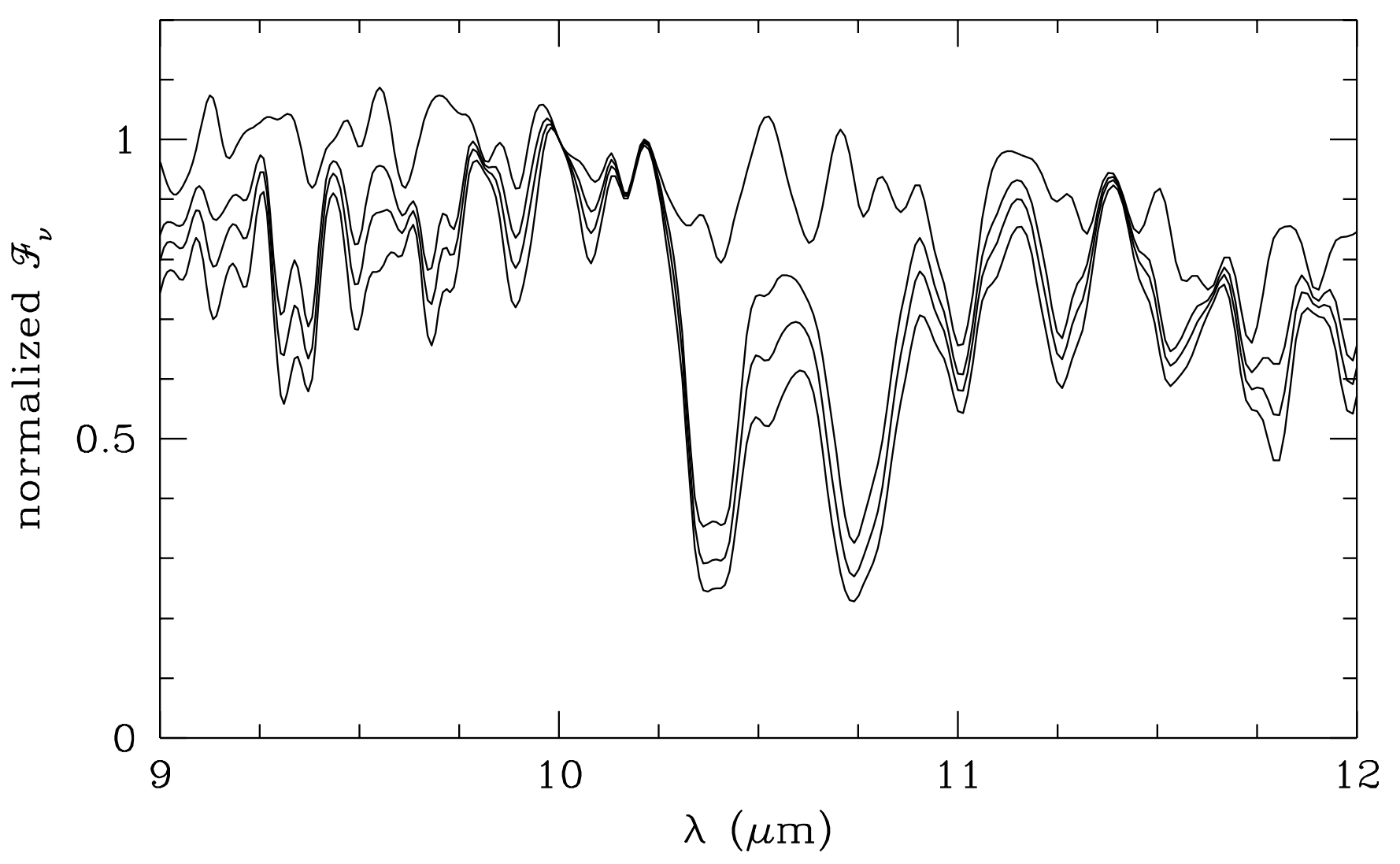

